\title{
HILL'S FORMULA FOR $g$-PERIODIC TRAJECTORIES OF LAGRANGIAN SYSTEMS
}

\author{
M. N. DAVLETSHIN
}

\begin{abstract}
In this paper some results of a work by Bolotin and Treshchëv are generalized to the case of $g$-periodic trajectories of Lagrangian systems. Formulae connecting the characteristic polynomial of the monodromy matrix with the determinant of the Hessian of the action functional are obtained both for the discrete and continuous cases. Applications to the problem of stability of $g$-periodic trajectories are given. Hill's formula can be used to study $g$-periodic orbits obtained by variational methods.
\end{abstract}

\section{$\S 1$. INTRODUCTION}

At the end of the 19th century the American astronomer and mathematician Hill [2, studying the nonautonomous second-order differential equation with periodic right-hand side

$$
\ddot{x}=a(t) x, \quad a(t+2 \pi)=a(t),
$$

obtained an expression for the multipliers of the monodromy matrix of a periodic solution in terms of the determinant of a certain infinite matrix $H$, elements of which depend on the Fourier coefficients of the right-hand side $a(t)$. Later Poincaré [3] explained in which sense the determinant of the infinite matrix should be understood, and proved its convergence. This result entered the theory of differential equations under the name of Hill's formula. Many years later, at the end of the 20th century, an analogue of Hill's formula for periodic solutions was found independently [4], [5] in the so-called discrete Lagrangian systems of differential equations. Here the analogue of the matrix $H$ is the finite Hessian matrix of the action functional at the critical point corresponding to a periodic solution. At approximately the same time, a generalization of Hill's formula for a periodic solution of an arbitrary continuous Lagrangian system appeared (see [6], 7]). Here, $H$ is the Hessian operator of the action functional at the critical point, which is defined by a periodic solution. Finally, in 2010 Bolotin and Treshchëv published the survey article [1], which to some extent summarizes all these results. Both versions of Hill's formula are considered, its applications to the stability of periodic solutions are discussed, and the case of degenerate trajectories is studied when Hill's formula degenerates. A connection between the dynamical stability of a periodic trajectory and the Morse index is given. It should be noted that the connection between the dynamical and geometric properties of a periodic trajectory (index, signature, and so on) was also studied from another viewpoint in [8], [10] and [11], [12]. The last paper uses an approach based on the methods of symplectic geometry.

In this paper we extend the results of [1] to the case of solutions which we call $g$ periodic solutions or trajectories in what follows. We now explain the aim of our paper. Let $g: M \rightarrow M$ be a diffeomorphism of the configuration manifold; $g$-periodic trajectories

2010 Mathematics Subject Classification. Primary 34D05; Secondary 37J25, 70H03.

Key words and phrases. Lagrangian systems, stability of $\boldsymbol{g}$-periodic trajectories. 
are defined to be the solutions of the Lagrangian system that have the following property:

$$
x_{n+i}=g x_{i} \quad \text { for any } i \in \mathbb{N}
$$

in the discrete case, and

$$
\gamma(t+\tau)=g \gamma(t) \quad \text { for any } t \in \mathbb{R}
$$

in the continuous case. In a situation where $g$ generates a cyclic group, we obtain a periodic trajectory. However, a $g$-periodic trajectory may not be periodic, for example, if $M$ is a two-dimensional plane and $g$ is a translation by a fixed vector. In what follows, $g$ is often an element of a continuous symmetry group of a Lagrangian system.

Thus, in the present paper we consider two types of Lagrangian systems:

- A discrete Lagrangian system (DLS) on a manifold $M$ with Lagrangian $L(x, y): M \times$ $M \rightarrow \mathbb{R}$, which is $g$-invariant, $L(g x, g y)=L(x, y)$, and satisfies some nondegeneracy condition. Then a $g$-periodic trajectory of period $n$ is a sequence $\mathbf{x}=\left(x_{i}\right)_{i \in \mathbb{Z}}$ satisfying condition (1.1) that is a critical point of the action functional on $M^{n}$ :

$$
\mathscr{A}(\mathbf{x})=\sum_{i=1}^{n} L\left(x_{i}, x_{i+1}\right), \quad x_{i+n}=g x_{i} .
$$

- A continuous Lagrangian system (CLS) with configuration manifold $M$ and a $\tau$ periodic Lagrangian $\mathscr{L}(x, \dot{x}, t)$ on $T M \times \mathbb{R}$ that is $g$-invariant, $\mathscr{L}(g(x), G(x) \dot{x}, t)=$ $\mathscr{L}(x, \dot{x}, t)$, where $G(x)$ is the differential of the map $g$ at the point $x$, and is strictly convex with respect to the velocity. Then a $g$-periodic trajectory $\gamma$ that satisfies condition (1.2) is a critical point of the action functional

$$
\mathscr{A}(\gamma)=\int_{0}^{\tau} \mathscr{L}(\gamma(t), \dot{\gamma}(t), t) d t
$$

on the set of $g$-periodic curves $\gamma: \mathbb{R} \rightarrow M$ of period $\tau$.

It is usually possible to reduce a discrete case to a continuous one, but it is more convenient to consider them separately.

We now present Hill's formula, which has the same form in both cases. Let $\widehat{G}^{-1} P$ be the monodromy matrix of the Poincaré map of a $g$-periodic trajectory, where $P$ is the operator of the period map, $h$ is the second variation of the action functional on the $g$-periodic trajectory, $H$ is the corresponding Hessian, and $G$ is the Jacobi matrix of the map $g$. Then

$$
\operatorname{det}\left(\widehat{G}^{-1} P-I\right)=\sigma(-1)^{m} \beta \operatorname{det} H,
$$

where $\widehat{G}$ is the Jacobi matrix of the map $\widehat{g}: M^{2} \rightarrow M^{2}, \widehat{g}(x, y)=(g(x), g(y)), x, y \in M$, $m=\operatorname{dim} M, \sigma=1$ for an orientable trajectory, and $\sigma=-1$ for a nonorientable trajectory (see details in 2.1 ). The coefficient $\beta$ is a positive factor. The sign of the expression $\sigma(-1)^{m} \operatorname{det} H$ is important for applications.

Formula (1.3) is the special case with $\rho=1$ of the formula

$$
\rho^{-m} \operatorname{det}\left(\widehat{G}^{-1} P-\rho I\right)=\sigma(-1)^{m} \beta \operatorname{det} H_{\rho}, \quad \rho \in \mathbb{C},
$$

where $H_{\rho}$ is the matrix that coincides with the Hessian matrix for $\rho=1$. The operator $\widehat{G}^{-1} P$ is symplectic, therefore both sides of (1.4) are polynomials of degree $m$ with respect to $\rho+\rho^{-1}$. A consequence of Hill's formula is that the geometric nondegeneracy of a $g$-periodic trajectory (the condition $\operatorname{det} H \neq 0$ ) and its dynamical nondegeneracy (the condition that 1 is an eigenvalue of $\widehat{G}^{-1} P$ ) are equivalent.

It should be noted that very similar objects in the continuous case were studied in [9]. The paper looked at solutions of the Hamiltonian system

$$
\dot{z}(t)=J H^{\prime}(t, z(t)), \quad S z(T)=z(0),
$$


where $S: \mathbb{R}^{2 n} \rightarrow \mathbb{R}^{2 n}$ is an orthogonal operator. An analogue of Hill's formula for these solutions was given, which is very similar to the Hill's formula we obtain here for $g$-periodic trajectories. Since $g$ preserves the Lagrangian, the operator $G: T_{\gamma(0)} M \rightarrow$ $T_{\gamma(\tau)} M$ is also orthogonal. Therefore some results in the 'continuous part' of our paper can be regarded as a Lagrangian version of the results in [9]. Furthermore, in [9] the important special case where $S^{m}=$ id was considered separately, and Hill's formula was given in this case. As in this paper, a connection between the linear stability of a trajectory and its Morse index was indicated.

The main area of application of Hill's formula is related to the stability of a $g$-periodic trajectory. If we have $\sigma(-1)^{m} \operatorname{det} H<0$, then the polynomial $F(\rho)=\operatorname{det}\left(\widehat{G}^{-1} P-\right.$ $\rho I)$ has a root greater than 1 . This implies that the $g$-periodic trajectory is unstable. In the nondegenerate situation $(\operatorname{det} H \neq 0)$ we have $\operatorname{sign}(\operatorname{det} H)=(-1)^{\text {ind } H}$, where ind $H$ is the Morse index of the $g$-periodic trajectory. Therefore, if a periodic trajectory is nondegenerate, then by (1.3) the inequality $\sigma(-1)^{m+i n d ~} H<0$ implies exponential instability.

A typical situation is when a $g$-periodic trajectory is degenerate. In the continuous case this happens, for example, in the autonomous case. In this case the variational equation has a $G$-periodic solution $\dot{\gamma}(t): \dot{\gamma}(t+\tau)=G(\gamma(t)) \dot{\gamma}(t)$. Hence the monodromy matrix has multiplier 1 and Hill's formula degenerates into the equation $0=0$. A trajectory can also degenerate because of the presence of a continuous symmetry group preserving the Lagrangian. This degeneracy generates $G$-periodic solutions and linear integrals of the variational equations. Equation (1.3) is useless for a degenerate periodic trajectory, since both sides of it vanish. Using the order reduction procedure it is possible to obtain a nondegenerate version of Hill's formula. Here we only consider the least degenerate case, when the Lie algebra $V$ of vector symmetry fields is commutative and the dimension of the generalized eigenspace $N$ for 1 of the operator $\widehat{G}^{-1} P$ is equal to $2 k$, where $k=\operatorname{dim} V$.

After eliminating all degeneracies we obtain the reduced Hill's formula, which looks similar, but the corresponding monodromy and Hessian operators $\widetilde{P}$ and $H^{\perp}$ act on smaller spaces:

$$
\operatorname{det}(\widetilde{P}-I)=\sigma^{\perp}(-1)^{m-k} \beta^{\perp} \operatorname{det} H^{\perp} .
$$

Here, $\sigma^{\perp} \in\{1,-1\}$ and $\beta^{\perp}>0$ are parameters of the reduced system.

To use the reduced Hill's formula in applications, it is necessary to understand the connection between $\sigma$ and $\sigma^{\perp}$, and also between ind $H$ and ind $H^{\perp}$, the Morse indices of the Hessians in the original and reduced systems. That is, given $\sigma$ and ind $H$, we must obtain $\sigma^{\perp}$ and ind $H^{\perp}$, which determine the stability of a trajectory. In $\S \S 3.4$ and 5.5 the main result is proved:

$$
\sigma(-1)^{\text {ind } H}=\sigma^{\perp}(-1)^{\text {ind } H^{\perp}+\text { ind } b},
$$

where the quadratic form $b$ on the generalized eigenspace $N=\operatorname{Ker}\left(\widehat{G}^{-1} P-I\right)^{2}$ is defined by the equation $b(v)=\omega\left(\left(\widehat{G}^{-1} P-I\right) v, v\right)$.

As an example, we consider autonomous Lagrangian systems that have no other degeneracies: $\operatorname{dim} V=1$ and $k=1$. A $g$-periodic trajectory $\gamma$ belongs to a smooth family of $g$-periodic trajectories. In this situation,

$$
(-1)^{\operatorname{ind} b}=-\operatorname{sign}\left(\frac{d E}{d \tau}\right)
$$

where $E$ and $\tau$ are the energy and period along this family. It follows from the reduced Hill's formula that if

$$
\sigma(-1)^{m+\text { ind } H} \frac{d E}{d \tau}<0
$$


then $\gamma$ has a real multiplier $\rho>1$. The sign of $d E / d \tau$ can be calculated, and as an example we do this for the problem of finding the motion of a point in $\mathbb{R}^{m}$ in a homogeneous potential force field (Example 5.22 ).

The paper consists of two parts: discrete and continuous. As already mentioned above, each case can be reduced to the other, but it makes sense to consider them separately. In $\S 2$ we first give the definition and basic properties of discrete Lagrangian systems (DLS). Then we prove Hill's formula for a $g$-periodic trajectory of a DLS (Theorem 2.15). In $\S 2.4$, as an application we give some sufficient conditions for periodic trajectories to be unstable. We devote special attention to studying the stability of billiard $g$-periodic trajectories of arbitrary dimension. It turns out that any $g$-periodic trajectory $\mathbf{x}$ of period $n$ of a billiard inside a hypersurface in $\mathbb{R}^{m+1}$ such that $(-1)^{m+n+\operatorname{ind}(\mathbf{x})}<0$ is exponentially unstable. We present several examples in $\S 2.5$

We next turn to studying the degenerate case where the monodromy matrix has eigenvalue 1 . We give the reduced Hill's formula and indicate a connection between the indices of the bilinear forms $h^{\perp}$ and $h$, which makes it possible to use the reduced Hill's formula in the problem of orbital stability. To do this we consider DLSs with symmetries in $\S 3$. Here many of the results are similar to the results in [1]; therefore we do not give the proofs of auxiliary assertions but simply state the results.

Section 4 is devoted to continuous Lagrangian systems. In this case the space of variations has infinite dimension, which makes the proof of Hill's formula somewhat more difficult. We present several versions of this formula similar to the discrete case. We indicate applications to the problem of the stability of $g$-periodic orbits in Lagrangian systems.

The main result of this part is: let $\gamma$ be a nondegenerate $g$-periodic geodesic on an $m$-dimensional manifold and suppose that $\sigma(-1)^{m+i n d} \gamma<0$. Then this geodesic is exponentially unstable (Assertion 4.35). Later on in the section we consider the case of orbit degeneracy. We present the reduced Hill's formula. As in the discrete case, we study the connection between the Morse index of a $g$-periodic trajectory of the original system and the corresponding solution of the reduced system and present applications of this formula to the problem of stability in Lagrangian systems with symmetry.

Finally, in $\$ 5.6$, as an example of a degenerate Lagrangian system, we consider an autonomous system - the motion of a point in $\mathbb{R}^{m}$ in a force field with homogeneous potential of degree $k$, where $k(k-2) \neq 0$. Here the main result is as follows: suppose that a $g$-periodic trajectory $\gamma$ has exactly 2 multipliers equal to 1 and $(-1)^{m+\text { ind } \gamma}(k-2) k<0$. Then $\gamma$ has a real multiplier $\rho>1$.

\section{§2. THE DISCRETE CASE}

2.1. Discrete Lagrangian systems (DLS). Let $M$ be an $m$-dimensional smooth manifold, and $L: M^{2} \rightarrow \mathbb{R}$ a smooth 1 function on $M^{2}=M \times M$. In what follows we use the notation

$$
\partial_{1} L(x, y)=\frac{\partial L(x, y)}{\partial x}, \quad \partial_{2} L(x, y)=\frac{\partial L(x, y)}{\partial y}, \quad B(x, y)=-\partial_{1} \partial_{2} L(x, y)
$$

${ }^{1} C^{2}$-smoothness is sufficient. 
In local coordinates,

$$
B(x, y)=\left(\begin{array}{ccc}
\frac{\partial^{2} L}{\partial y_{1} \partial x_{1}} & \cdots & \frac{\partial^{2} L}{\partial y_{1} \partial x_{m}} \\
\cdots \ldots \ldots \ldots & \ldots \ldots \ldots \ldots \\
\frac{\partial^{2} L}{\partial y_{m} \partial x_{1}} & \cdots & \frac{\partial^{2} L}{\partial y_{m} \partial x_{m}}
\end{array}\right)
$$

We call $L$ a discrete Lagrangian if the following condition holds.

Twisting condition. $B(x, y)$ is nondegenerate for all $x, y \in M$.

Remark 2.1. For our purposes it is sufficient that the nondegeneracy condition holds in some neighborhood of the trajectory under consideration.

Any discrete Lagrangian $L$ determines the map

$$
T: M^{2} \rightarrow M^{2}, \quad T(x, y)=(y, z),
$$

locally, where the point $z=z(x, y)$ is defined by the equation

$$
\frac{\partial}{\partial y}(L(x, y)+L(y, z))=\partial_{2} L(x, y)+\partial_{1} L(y, z)=0
$$

By the implicit function theorem the twisting condition allows us to give a unique local expression for the map $z=z(x, y)$. In the general situation, $T$ is a multi-valued map with the graph

$$
\Gamma=\left\{(x, y, y, z) \in M^{2} \times M^{2}: \partial_{2} L(x, y)+\partial_{1} L(y, z)=0\right\} .
$$

More details about systems with a multi-valued Lagrangian can be found in [13.

The dynamical system defined by the map $T$ is called a discrete Lagrangian system (DLS) with configuration space $M$ and Lagrangian $L$.

Remark 2.2. A discrete Lagrangian is determined up to multiplication by a nonzero constant, up to adding a constant, and up to a gauge transformation

$$
L(x, y) \mapsto L(x, y)+f(x)-f(y),
$$

where $f: M \rightarrow \mathbb{R}$ is a smooth function.

Billiards give an important example of DLSs.

Definition 2.3. Let $M$ be a smooth convex hypersurface in $\mathbb{R}^{m+1}$. A billiard is defined to be a DLS with the Lagrangian $L(x, y)=|x-y|$.

One can show that in this case the bilinear form $B(x, y): T_{x} M \rightarrow T_{y}^{*} M$ acts by the rule

$$
\langle B(x, y) v, w\rangle=\frac{\langle v, w\rangle-\langle v, e\rangle\langle w, e\rangle}{|x-y|}, \quad e=\frac{x-y}{|x-y|} .
$$

Assertion 2.4. We have $\operatorname{det} B(x, y)<0$.

Proof. Suppose that $M$ is oriented as the boundary of the domain bounded by it. Let $\Pi(x, y): T_{x} M \rightarrow T_{y} M$ be given by $\Pi v=v+x-y$ for $v \in T_{x} M$. Then

$$
\langle B(x, y) v, \Pi(x, y) v\rangle=\frac{|v|^{2}-\langle v, e\rangle^{2}}{|x-y|}>0 \quad \text { for all } v \in T_{x} M \backslash\{0\} .
$$

The map $\Pi(x, y)$ changes orientation; consequently, $\operatorname{det} B(x, y)<0$.

Remark 2.5. Unlike its sign, the determinant of the matrix of the bilinear form $B(x, y)$ is not uniquely determined.

For more details about DLSs see [13] and [14]. 
2.2. $g$-periodic trajectories. Let $g: M \rightarrow M$ be a diffeomorphism of the configuration manifold onto itself. We say that $g$ preserves the Lagrangian $L$ if $L(g x, g y)=L(x, y)$ for any $x, y \in M$. We also denote the differential of $g$ at the point $x$ by $D g(x)$.

Definition 2.6. A sequence of points $\left(x_{i}\right)_{i \in \mathbb{Z}} \subset M$ is said to be $g$-periodic with period $n$ if $x_{i+n}=g x_{i}$ for any $i$.

Let $\mathbf{x}=\left(x_{i}\right)_{i \in \mathbb{Z}}$ be a $g$-periodic sequence. We define the action functional on the class of $g$-periodic sequences $\mathbf{x}$ on $M$ :

$$
\mathscr{A}(\mathbf{x}) \stackrel{\text { def }}{=} L\left(x_{1}, x_{2}\right)+L\left(x_{2}, x_{3}\right)+\cdots+L\left(x_{n}, g x_{1}\right) .
$$

Remark 2.7. The action functional is well defined, since $\mathscr{A}(\mathbf{x})$ is independent of the choice of $n$ consecutive points of the trajectory $\mathbf{x}$.

Proof. We verify that $\mathscr{A}(\mathbf{x})$ does not change under a shift of the segment $x_{1}, \ldots, x_{n}$. Let

$$
\mathscr{B}(\mathbf{x})=L\left(x_{2}, x_{3}\right)+L\left(x_{2}, x_{3}\right)+\cdots+L\left(x_{n}, g x_{1}\right)+L\left(g x_{1}, g x_{2}\right) .
$$

Then

$$
\mathscr{A}(\mathbf{x})-\mathscr{B}(\mathbf{x})=L\left(x_{1}, x_{2}\right)-L\left(g x_{1}, g x_{2}\right)=0,
$$

since $g$ preserves the Lagrangian.

Assertion 2.8. A g-periodic sequence $\mathbf{x}$ is a trajectory of the DLS if and only if $\mathbf{x}$ is a critical point of the functional $\mathscr{A}(\mathbf{x})$.

Proof. Let $\mathbf{x}=\left(x_{i}\right)_{i \in \mathbb{Z}}$ be a critical point of the functional, that is, $\partial \mathscr{A}(\mathbf{x}) / \partial x_{i}=0$ for all $i=1, \ldots, n$. Then

$$
\begin{gathered}
\frac{\partial L\left(x_{1}, x_{2}\right)}{\partial x_{1}}+\frac{\partial L\left(x_{n}, g x_{1}\right)}{\partial\left(g x_{1}\right)} D g\left(x_{1}\right)=0 \\
\frac{\partial L\left(x_{i-1}, x_{i}\right)}{\partial x_{i}}+\frac{\partial L\left(x_{i}, x_{i+1}\right)}{\partial x_{i}}=0, \quad i=2, \ldots, n, \quad x_{n+1}=g x_{1} .
\end{gathered}
$$

By transforming the first expression to

$$
\begin{aligned}
\frac{\partial L\left(x_{1}, x_{2}\right)}{\partial x_{1}}+\frac{\partial L\left(x_{n}, g x_{1}\right)}{\partial x_{1}} & =\frac{\partial L\left(g x_{1}, g x_{2}\right)}{\partial x_{1}}+\frac{\partial L\left(x_{n}, g x_{1}\right)}{\partial x_{1}} \\
& =\left(\partial_{2} L\left(x_{n}, x_{n+1}\right)+\partial_{1} L\left(x_{n+1}, x_{n+2}\right)\right) D g\left(x_{1}\right)=0,
\end{aligned}
$$

we see that $\left(x_{i}\right)_{i \in \mathbb{Z}}$ is a trajectory of the DLS.

We define the map

$$
\widehat{g}: M^{2} \rightarrow M^{2}, \quad \widehat{g}(a) \stackrel{\text { def }}{=}\left(g x_{0}, g x_{1}\right) \in M^{2}, \quad a=\left(x_{0}, x_{1}\right) \in M^{2} .
$$

Let $x=\left(x_{1}, \ldots, x_{n}\right) \in M^{n}$ be $n$ consecutive points of a $g$-periodic trajectory $\left(x_{i}\right)_{i \in \mathbb{Z}}$. We define $\mathbf{H}=\partial^{2} \mathscr{A}(\mathbf{x}) / \partial x^{2}$ to be the Hessian of the action at the point $\mathbf{x} \in M^{n}$. We denote the differentials of the maps $T: M^{2} \rightarrow M^{2}$ and $T^{n}: M^{2} \rightarrow M^{2}$ by

$$
\begin{gathered}
P_{i}=D T\left(x_{i-1}, x_{i}\right), \quad x_{0}=g^{-1} x_{n}, \\
P=D T^{n}(a): T_{a} M^{2} \rightarrow T_{\widehat{g} a} M^{2} .
\end{gathered}
$$

The explicit form of the Hessian of the action is

$$
\mathbf{H}=\left(\begin{array}{ccccc}
A_{1} & -B_{1}^{*} & 0 & \ldots & -G^{*}\left(x_{1}\right) B_{n} \\
-B_{1} & A_{2} & -B_{2}^{*} & \ldots & 0 \\
0 & -B_{2} & A_{3} & \ldots & 0 \\
\ldots \ldots \ldots \ldots \ldots \ldots \ldots \ldots \ldots \ldots \ldots \ldots \ldots \ldots \ldots \ldots \ldots & \ldots \ldots \ldots
\end{array}\right) .
$$


Here, the $A_{i}, B_{i}$ are $m \times m$ matrices:

$$
\begin{aligned}
& A_{i}=\frac{\partial^{2} \mathscr{A}(\mathbf{x})}{\partial x_{i}^{2}}=\partial_{2} \partial_{2} L\left(x_{i-1}, x_{i}\right)+\partial_{1} \partial_{1} L\left(x_{i}, x_{i+1}\right), \quad i=1, \ldots, n, \\
& B_{i}=B\left(x_{i}, x_{i+1}\right), \quad i=1, \ldots, n, x_{n+1}=g x_{1}, \\
& G \text { is the Jacobian matrix of the map } g .
\end{aligned}
$$

By the chain rule,

$$
\frac{\partial^{2} L(x, g y)}{\partial x \partial y}=G^{*}(y) \partial_{1} \partial_{2} L(x, g y) .
$$

It is also easy to verify that on a $g$-periodic trajectory we have

$$
\frac{\partial^{2} L\left(x_{1}, x_{2}\right)}{\partial x_{1}^{2}}+\frac{\partial^{2} L\left(x_{n}, g x_{1}\right)}{\partial x_{1}^{2}}=G\left(x_{1}\right)^{*}\left(\partial_{1} \partial_{1} L\left(g x_{1}, g x_{2}\right)+\partial_{2} \partial_{2} L\left(x_{n}, g x_{1}\right)\right) G\left(x_{1}\right) .
$$

Definition 2.9. Let $\mathbf{u}=\left(u_{1}, \ldots, u_{n}\right)$, where $u_{i} \in T_{x_{i}} M, i=1, \ldots, n, u_{n+i}=G u_{i}$. We call the system $\mathbf{H u}=0$ the system of variational equations.

Assertion 2.10. A vector $\mathbf{u}$ satisfies the system of variational equations if and only if $P_{i}\left(u_{i-1}, u_{i}\right)=\left(u_{i}, u_{i+1}\right)$,

$$
u_{0} \in T_{x_{0}} M, \quad u_{n+1} \in T_{x_{n+1}} M, \quad u_{0}=\left(G\left(x_{0}\right)\right)^{-1} u_{n}, \quad u_{n+1}=G\left(x_{1}\right) u_{1} .
$$

Proof. We calculate $A_{1}, A_{i}, i=2, \ldots, n$, and $B_{i}$ explicitly:

$$
\begin{aligned}
& A_{1}=\left(G\left(x_{1}\right)\right)^{*}\left(\partial_{1} \partial_{1} L\left(g x_{1}, g x_{2}\right)+\partial_{2} \partial_{2} L\left(x_{n}, g x_{1}\right)\right) G\left(x_{1}\right), \\
& A_{i}=\partial_{1} \partial_{1} L\left(x_{i-1}, x_{i}\right)+\partial_{2} \partial_{2} L\left(x_{i}, x_{i+1}\right), \quad i=2, \ldots, n, \\
& B_{i}=B\left(x_{i}, x_{i+1}\right)=-\partial_{1} \partial_{2} L\left(x_{i}, x_{i+1}\right) .
\end{aligned}
$$

We write the condition $\mathbf{H u}=0$ :

$$
\begin{aligned}
A_{1} u_{1}-B_{1}^{*} u_{2}-G^{*}\left(x_{1}\right) B_{n} u_{n}=0 & \text { for } i=1, \\
-B_{i-1} u_{i-1}+A_{i} u_{i}-B_{i+1}^{*} u_{i+1}=0 & \text { for } i=2, \ldots, n-1, \\
-B_{n}^{*} G\left(x_{1}\right) u_{1}-B_{n-1} u_{n-1}+A_{n} u_{n}=0 & \text { for } i=n .
\end{aligned}
$$

We rewrite the last equation in the form

$$
-B^{*} u_{n+1}-B_{n-1} u_{n-1}+A_{n} u_{n}=0, \quad u_{n+1}=G\left(x_{1}\right) u_{1} .
$$

We need to consider three separate cases: $i=1,2 \leq i \leq n-1$, and $i=n$. For example, consider the case $i=1$. In the other cases the arguments are similar. We have

$$
P_{1}=D T\left(x_{0}, x_{1}\right), \quad T\left(x_{0}, x_{1}\right)=\left(x_{1}, x_{2}\right) .
$$

Then the matrix $P_{1}$ has the form

$$
\left(\begin{array}{ll}
\frac{\partial x_{1}}{\partial x_{0}} & \frac{\partial x_{1}}{\partial x_{1}} \\
\frac{\partial x_{2}}{\partial x_{0}} & \frac{\partial x_{2}}{\partial x_{1}}
\end{array}\right)=\left(\begin{array}{cc}
0 & I \\
\frac{\partial x_{2}}{\partial x_{0}} & \frac{\partial x_{2}}{\partial x_{1}}
\end{array}\right)
$$

We find $\partial x_{2} / \partial x_{0}$ and $\partial x_{2} / \partial x_{1}$ from the equation

$$
\frac{\partial L\left(x_{0}, x_{1}\right)}{\partial x_{1}}+\frac{\partial L\left(x_{1}, x_{2}\right)}{\partial x_{1}}=0 .
$$

Recall that if $F\left(x_{0}, x_{1}, x_{2}\right)=0$, then

$$
\frac{\partial x_{2}}{\partial x_{0}}=-{\frac{\partial F}{\partial x_{2}}}^{-1} \frac{\partial F}{\partial x_{0}}, \quad \frac{\partial x_{2}}{\partial x_{1}}=-{\frac{\partial F}{\partial x_{2}}}^{-1} \frac{\partial F}{\partial x_{1}} .
$$


Consequently,

Then

$$
\begin{aligned}
\frac{\partial F}{\partial x_{2}} & =\frac{\partial}{\partial x_{2}} \frac{\partial L\left(x_{1}, x_{2}\right)}{\partial x_{1}}=\partial_{2} \partial_{1} L\left(x_{1}, x_{2}\right)=-B_{1}^{*}, \\
\frac{\partial F}{\partial x_{0}} & =\frac{\partial}{\partial x_{0}} \frac{\partial L\left(g x_{0}, g x_{1}\right)}{\partial x_{1}}=-G^{*}\left(x_{1}\right) B_{n} G\left(x_{0}\right), \\
\frac{\partial F}{\partial x_{1}} & =\frac{\partial}{\partial x_{1}} \frac{\partial L\left(x_{0}, x_{1}\right)}{\partial x_{1}}+\frac{\partial}{\partial x_{1}} \frac{\partial L\left(x_{1}, x_{2}\right)}{\partial x_{1}} \\
& =\frac{\partial}{\partial x_{1}} \frac{\partial L\left(g x_{0}, g x_{1}\right)}{\partial x_{1}}+\frac{\partial}{\partial x_{1}} \frac{\partial L\left(x_{0}, x_{1}\right)}{\partial x_{1}}=A_{1} .
\end{aligned}
$$

$$
\begin{aligned}
P_{1}\left(u_{0}, u_{1}\right) & =\left(\begin{array}{cc}
0 & I \\
-\left(B_{1}^{*}\right)^{-1} G^{*}\left(x_{1}\right) B_{n} G\left(x_{0}\right) & \left(B_{1}^{*}\right)^{-1} A_{1}
\end{array}\right)\left(\begin{array}{l}
u_{0} \\
u_{1}
\end{array}\right) \\
& =\left(\begin{array}{c}
u_{1} \\
\left(B_{1}^{*}\right)^{-1}\left(-G^{*}\left(x_{1}\right) B_{n} G\left(x_{0}\right) u_{0}+A_{1} u_{1}\right)
\end{array}\right) \\
& =\left(\begin{array}{c}
u_{1} \\
\left(B_{1}^{*}\right)^{-1}\left(-G^{*}\left(x_{1}\right) B_{n} u_{n}+A_{1} u_{1}\right)
\end{array}\right) .
\end{aligned}
$$

Consequently,

$$
u_{2}=\left(B_{1}^{*}\right)^{-1}\left(-G^{*}\left(x_{1}\right) B_{n} u_{n}+A_{1} u_{1}\right)
$$

and this means that $P_{1}\left(u_{0}, u_{1}\right)=\left(u_{1}, u_{2}\right)$.

Corollary 2.11. The equation $\mathbf{H u}=0$ holds if and only if $P\left(u_{1}, u_{2}\right)=\widehat{G}\left(u_{1}, u_{2}\right)$, where $\widehat{G}\left(u_{1}, u_{2}\right) \stackrel{\text { def }}{=}\left(G u_{1}, G u_{2}\right)$.

Proof. We have

$$
P \stackrel{\text { def }}{=} D T^{n}(a) \Longrightarrow P(a)=D T\left(x_{0}, x_{1}\right) \circ D T\left(x_{1}, x_{2}\right) \circ \cdots \circ D T\left(x_{n-1}, x_{n}\right) .
$$

Therefore this assertion follows from the preceding lemma.

2.3. Sequences which are $g$-quasiperiodic. From here on $T_{x_{i}} M$ will denote its complexification. We now define a generalization of the Hessian $\mathbf{H}$.

Definition 2.12. Let $S^{1}=\{\rho \in \mathbb{C}:|\rho|=1\}$. A sequence $u_{i} \in T_{x_{i}} M$ is said to be $g$-quasiperiodic if $u_{n+1}=\rho G\left(x_{1}\right) u_{i}$, where $\rho \in S^{1}$.

Let $\left(u_{j}\right)_{j \in \mathbb{Z}}$ be a $g$-quasiperiodic vector sequence satisfying the condition $P_{i}\left(u_{i-1}, u_{i}\right)=$ $\left(u_{i}, u_{i+1}\right)$. Then $\mathbf{u}$ satisfies the following system of variational equations:

$$
\left\{\begin{array}{l}
A_{1} u_{1}-B_{1}^{*} u_{2}-\rho^{-1} G^{*}\left(x_{1}\right) B_{n} u_{n}=0 \\
-B_{i-1} u_{i-1}+A_{i} u_{i}-B_{i}^{*} u_{i+1}=0, \quad i=2, \ldots, n-1, \\
-\rho B_{n}^{*} u_{n+1}-B_{n-1} u_{n-1}+A_{n} u_{n}=0 .
\end{array}\right.
$$

The $\rho$-Hessian $\mathbf{H}_{\rho}$ corresponds to this system and has the form

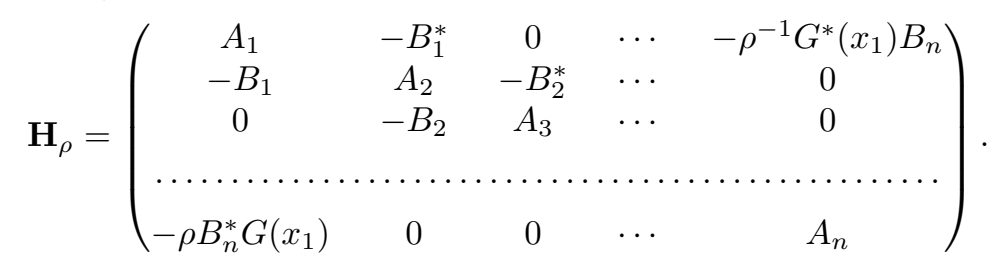

Assertion 2.13. Suppose that $\mathbf{H}_{\rho} \mathbf{u}=0$. Then $P_{i}\left(u_{i-1}, u_{i}\right)=\left(u_{i}, u_{i+1}\right), i=1, \ldots n$. 
The proof is similar to the proof of Assertion 2.10.

Corollary 2.14. If $\mathbf{H}_{\rho} \mathbf{u}=0$, then $P\left(u_{1}, u_{2}\right)=\left(\rho G u_{1}, \rho G u_{2}\right)=\rho \widehat{G}\left(u_{1}, u_{2}\right)$.

Theorem 2.15 (Hill's formula). For any $\rho \in \mathbb{C} \backslash\{0\}$,

$$
\rho^{-m} \operatorname{det}\left(\widehat{G}^{-1}(a) P-\rho I\right)=\frac{(-1)^{m} \operatorname{det} \mathbf{H}_{\rho}}{\operatorname{det} G^{2}\left(x_{1}\right) \prod_{i=1}^{n} \operatorname{det} B_{i}} .
$$

Proof. Both sides of (2.4) are polynomials of degree $m$ with respect to $\rho+\rho^{-1}$. Let

$$
E(\rho)=\operatorname{det}\left(\widehat{G}^{-1}(a) P-\rho I\right) \operatorname{det} G^{2}\left(x_{1}\right), \quad F(\rho)=(-1)^{m} \rho^{m} \frac{\operatorname{det} \mathbf{H}_{\rho}}{\prod_{i=1}^{n} \operatorname{det} B_{i}} .
$$

These are polynomials of degree $2 m$ with respect to $\rho$. It follows from Corollary 2.14 that $\operatorname{det}\left(\widehat{G}^{-1}(a) P-\rho I\right)=0$ is equivalent to $\operatorname{det} \mathbf{H}_{\rho}=0$, and so they have the same roots. We claim that the leading terms of $E(\rho)$ and $F(\rho)$ coincide.

The coefficient of the highest power of $E(\rho)$ is equal to $\operatorname{det} G^{2}\left(x_{1}\right)$, since the matrix $P$ has size $2 m \times 2 m$. In coordinates, $\mathbf{H}_{\rho}$ is an $m n \times m n$ matrix that coincides with $\mathbf{H}$ with two exceptions: in the right upper $m \times m$ block $-G^{*}\left(x_{1}\right) B_{n}$ is replaced by $-\rho G^{*}\left(x_{1}\right) B_{n}$, and in the left lower block of size $m \times m$ the matrix $-B^{*} G\left(x_{1}\right)$ is replaced by $-\rho B^{*} G\left(x_{1}\right)$. Consequently, the coefficient of the highest power of the polynomial $F(\rho)$ is equal to

$$
\begin{aligned}
&(-1)^{m}(-1)^{m^{2} n}(-1)^{m(n-1)} \prod_{i=1}^{n} \operatorname{det} B_{i} \operatorname{det} G^{2}\left(x_{1}\right)\left(\prod_{i=1}^{n} \operatorname{det} B_{i}\right)^{-1} \\
&=(-1)^{m n(m+1)} \operatorname{det} G^{2}\left(x_{1}\right)=\operatorname{det} G^{2}\left(x_{1}\right) .
\end{aligned}
$$

Thus, the leading terms of the polynomials $E(\rho)$ and $F(\rho)$ coincide. Thus, the polynomials $E(\rho)$ and $F(\rho)$ have the same degree, roots and leading terms, and therefore they coincide.

Corollary 2.16. For $\rho=1$ we have

$$
\begin{gathered}
\operatorname{det}\left(\widehat{G}^{-1}(a) P-I\right) \operatorname{det} G^{2}\left(x_{1}\right)=\sigma(\mathbf{x})(-1)^{m} \beta \operatorname{det} \mathbf{H}, \\
\sigma(\mathbf{x})=\operatorname{sign}\left(\prod_{i=1}^{n} \operatorname{det} B_{i}\right), \quad \beta=\left|\prod_{i=1}^{n} \operatorname{det} B_{i}\right|^{-1} .
\end{gathered}
$$

2.4. Some applications of Hill's formula. Let ind $\mathbf{H}$ denote the Morse index of $\mathscr{A}$ at a critical point $\mathbf{x}$, in other words, the dimension of the negative subspace of the quadratic form $\mathbf{H}$.

Corollary 2.17. It follows from (2.5) that

$$
\operatorname{det}\left(\widehat{G}^{-1}(a) P-I\right)=0 \text { if and only if } \operatorname{det} \mathbf{H}=0 .
$$

Thus, if the determinants do not equal zero, then by continuity they both remain nonzero under a small deformation of the orbit. This means that under a small deformation of $T$ a nondegenerate $g$-periodic trajectory does not vanish and ind $\mathbf{H}$ remains the same.

Now suppose that both sides of formula (2.4) are nonzero.

Corollary 2.18. It follows from (2.5) that

$$
\sigma(\mathbf{x})(-1)^{m+\operatorname{ind} \mathbf{H}} \operatorname{det}\left(\widehat{G}^{-1}(a) P-I\right)>0 .
$$

Definition 2.19. We say that a $g$-periodic trajectory is linearly stable if a fixed point of the map $\widehat{g}^{-1} \circ T^{n}: M^{2} \rightarrow M^{2}$ is stable in the linear approximation. 
Definition 2.20. The matrix $G^{-1}(a) P$ is called the monodromy matrix, and its eigenvalues are called the multipliers of the $g$-periodic trajectory.

Assertion 2.21. If $\operatorname{det}\left(\widehat{G}^{-1}(a) P-I\right)<0$, then there exists a real multiplier $\rho>1$.

Proof. Consider the characteristic polynomial

$$
F(\rho)=\operatorname{det}\left(\widehat{G}^{-1}(a)-\rho I\right) .
$$

Since $F(\rho)$ is a continuous function and $P$ is an even-dimensional matrix, we have $F(+\infty)=+\infty$ and $F(1)<0$. Consequently, there exists $\rho^{*} \in(1,+\infty)$ such that $F\left(\rho^{*}\right)=0$.

Corollary 2.22. In the case $\sigma(\mathbf{x})(-1)^{m+i n d} \mathbf{H}<0$, the trajectory $\mathbf{x}$ is unstable: there exists a real multiplier $\rho>1$.

Corollary 2.23. Suppose that $\sigma(\mathbf{x})(-1)^{m+n}<0$ and $\mathbf{x}$ is a nondegenerate local maximum of the action $\mathscr{A}$. Then $\mathbf{x}$ is dynamically unstable.

Corollary 2.24. Suppose that $\sigma(\mathbf{x})(-1)^{m}<0$ and $\mathbf{x}$ is a nondegenerate local minimum of the action $\mathscr{A}$. Then $\mathbf{x}$ is dynamically unstable: there exists a real multiplier $\rho>1$.

It follows from Assertion 2.4 that $\sigma(\mathbf{x})=(-1)^{n}$ for a billiard trajectory.

Corollary 2.25. Suppose that a g-periodic billiard trajectory of period $n$ is such that $(-1)^{m+n+\mathrm{ind} \mathbf{H}}<0$. Then the billiard trajectory is unstable.

2.5. Examples. We illustrate the applications of Hill's formula for $g$-periodic solutions by examples.

Example 2.26. Consider a cylindrical surface with mirrored inner surface which has periodic structure along the axis. A ray of light enters this tube and is reflected inside it by the law the angle of incidence equals the angle of reflection. One can show that this condition is equivalent to the fact that a sequence of reflection points is a trajectory of a discrete Lagrangian system with the Lagrangian $L(x, y)=|x-y|$. As a result we obtain a billiard. Suppose that the reflection points of the ray form a $g$-periodic sequence of period $n$. Using Hill's formula, in a number of cases it is possible to prove that such a billiard trajectory is unstable. As a model example we consider a planar billiard in a band with periodic structure whose boundary is defined by the following equations in a Cartesian system of coordinates $(x, y)$ :

$$
y_{1}=h-\cos x, \quad y_{2}=-h-\cos x, \quad h>1 .
$$

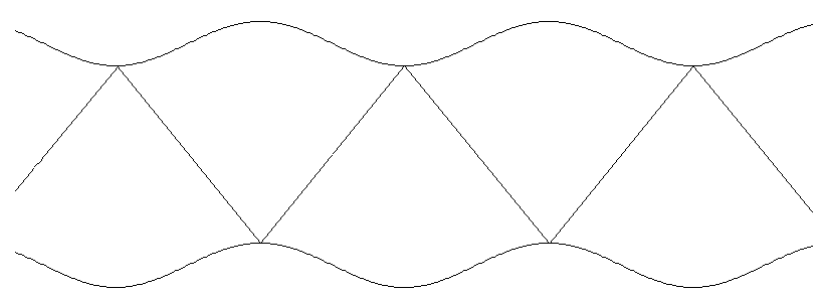

Figure 1

We shall examine the stability of the $g$-periodic trajectory $\ldots,(-2 \pi ; h-1),(-\pi ; 1-h)$, $(0 ; h-1),(\pi ; 1-h),(2 \pi ; h-1), \ldots$. 
Here, $g$ is the translation along the $x$-axis by $\pi$ and $n=2$. In this case the Hessian matrix takes the form

$$
\mathbf{H}=\left(\begin{array}{cc}
\frac{(2 h-2)^{2}+(2 h-2)^{3}}{\left(\pi^{2}+(2 h-2)^{2}\right)^{3 / 2}} & -\frac{(2 h-2)^{2}}{\left(\pi^{2}+(2 h-2)^{2}\right)^{3 / 2}} \\
-\frac{(2 h-2)^{2}}{\left(\pi^{2}+(2 h-2)^{2}\right)^{3 / 2}} & \frac{(2 h-2)^{2}+(2 h-2)^{3}}{\left(\pi^{2}+(2 h-2)^{2}\right)^{3 / 2}}
\end{array}\right) .
$$

Since $h>1$, it follows that ind $\mathbf{H}=0$. Then $(-1)^{m+n+\operatorname{ind} \mathbf{H}}=(-1)^{1+2}<0$; hence the trajectory is unstable.

Example 2.27. Hill's formula for $g$-periodic trajectories can also be useful for studying the stability of periodic trajectories when Hill's formula for periodic trajectories cannot help. This can happen, for example, if a billiard trajectory has symmetry. The fact is that the period and index of a periodic trajectory and of the corresponding $g$-periodic trajectory are different. Let ind $\mathbf{H}_{\text {per. }}$ denote the Morse index of the periodic trajectory, and ind $\mathbf{H}_{g}$ the Morse index of the $g$-periodic trajectory, where $g$ is a symmetry of the billiard trajectory, $n$ is the period of the periodic trajectory, and $k$ of the $g$-periodic one. Then a situation is possible where $(-1)^{m+n+\text { ind }} \mathbf{H}_{\text {per. }}=1$ and $(-1)^{m+k+\text { ind } \mathbf{H}_{g}}=-1$.

\section{§3. Degeneracy in Hill's formula}

Hill's formula provides nontrivial information in the case when a $g$-periodic trajectory is nondegenerate. In this section we consider the degenerate case, present a reduced version of Hill's formula, and make it applicable to solving the stability problem.

The results of this section are similar to the results of the corresponding chapter in [1]; therefore we shall not give the proofs of the auxiliary results, but just state them.

3.1. Symplectic reduction for the Poincaré map. Suppose that a $g$-periodic trajectory is degenerate. Then the linear Poincaré map $\widehat{G}^{-1} P: W \rightarrow W$ has eigenvalue 1: there exists $w \neq 0$ such that $P w=\widehat{G} w$. Since $\widehat{G}^{-1} P$ is symplectic, we have $\omega\left(w, \widehat{G}^{-1} P u\right)=$ $\omega(w, u)$, so that $K_{w}(u)=\omega(w, u)$ is a linear first integral of $\widehat{G}^{-1} P$. Then $\widehat{G}^{-1} P$ can be reduced to a linear symplectic map $\widetilde{P}: \widetilde{W} \rightarrow \widetilde{W}$ of lower dimension. Suppose that there are several eigenvectors corresponding to the eigenvalue 1. Let $V \subset\left\{w \in W: \widehat{G}^{-1} P w=w\right\}$. Then $\widehat{G}^{-1} P$ has the first integral $K: W \rightarrow V^{*}$ : for $w \in V$ we obtain $\langle K(u), w\rangle=K_{w}(u)$. In what follows we assume that $V$ is isotropic: $\left.\omega\right|_{V}=0$. Then $V \subset K^{-1}(0)$. We set $\widetilde{W}=K^{-1}(0) / V$.

Proposition 3.1. The operator $\widehat{G}^{-1} P$ generates a reduced symplectic operator $\widetilde{P}: \widetilde{W} \rightarrow$ $\widetilde{W}$ such that the diagram

is commutative. Moreover,

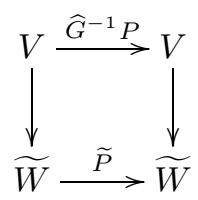

$$
\operatorname{det}\left(\widehat{G}^{-1} P-\rho I_{W}\right)=(1-\rho)^{2 k} \operatorname{det}\left(\widetilde{P}-\rho I_{\widetilde{W}}\right), \quad k=\operatorname{dim} V .
$$

3.2. Routh order reduction for linear discrete Lagrangian systems. We now translate Proposition 3.1 into the language of the system of variational equations, that is, the linear Lagrangian system $(E, \Lambda)$. To every eigenvector $w$ of the Poincaré map $\widehat{G}^{-1} P$ there corresponds a nonzero $G$-periodic solution $\mathbf{w}=\left(w_{i}\right)$ of the variational equations. 
Assertion 3.2. The solution $\mathbf{w}$ has the corresponding linear first integral $I_{j}\left(u_{j}, u_{j+1}\right)=$ $\left\langle B_{j} w_{j}, u_{j+1}\right\rangle-\left\langle B_{j} u_{j}, w_{j+1}\right\rangle$.

Indeed, if $\mathbf{u}=\left(u_{j}\right)$ is a solution of the system $\mathbf{H u}=0$, then

$$
\begin{array}{r}
0=\left\langle A_{1} w_{1}-B_{1} w_{2}-G^{*}\left(x_{1}\right) B_{n} w_{0}, u_{1}\right\rangle-\left\langle A_{1} u_{1}-B_{1}^{*} u_{2}-B_{n}^{*} G\left(x_{1}\right) u_{0}, w_{1}\right\rangle \\
=I_{1}\left(u_{1}, u_{2}\right)-I_{0}\left(u_{0}, u_{1}\right), \\
\begin{array}{r}
0=\left\langle A_{j} w_{j}-B_{j}^{*} w_{j+1}-B_{j-1} w_{j-1}, u_{j}\right\rangle-\left\langle A_{j} u_{j}-B_{j}^{*} u_{j+1}-B_{j-1} u_{j-1}, w_{j}\right\rangle \\
=I_{j}\left(u_{j}, u_{j+1}\right)-I_{j-1}\left(u_{j-1}, u_{j}\right), \quad j=2, \ldots, n-1 .
\end{array}
\end{array}
$$

Thus,

$$
I_{j-1}\left(u_{j-1}, u_{j}\right)=I_{j}\left(u_{j}, u_{j+1}\right) .
$$

In fact, $I_{j}\left(u_{j}, u_{j+1}\right)=K_{w}(u)$, where $K_{w}$ is an integral of the Poincaré map and $u \in W$ corresponds to the trajectory $\left(u_{j}\right)$.

We now suppose that there are several eigenvectors and $V \subset \operatorname{Ker}\left(\widehat{G}^{-1} P-I\right)$ is an isotropic subspace. Let $\Gamma \subset X$ denote the space of $G$-periodic trajectories corresponding to $V$. Let $w^{\alpha} \in V, \alpha=1, \ldots, k$, be a basis in $V$. Then the corresponding $G$-periodic trajectories $\mathbf{w}^{\alpha}=\left(w_{i}^{\alpha}\right)$ form a basis in $\Gamma$. Let

$$
I_{j}^{\alpha}\left(u_{j}, u_{j+1}\right)=\left\langle B_{j} w_{j}^{\alpha}, u_{j+1}\right\rangle-\left\langle B_{j} u_{j}, w_{j+1}^{\alpha}\right\rangle
$$

be the corresponding integrals of the variational equations. Since $V$ is isotropic, the integrals commute:

$$
I_{j}^{\alpha}\left(w_{j}^{\beta}, w_{j+1}^{\beta}\right)=\left\langle B_{j} w_{j}^{\alpha}, w_{j+1}^{\beta}\right\rangle-\left\langle B_{j} w_{j}^{\beta}, w_{j+1}^{\alpha}\right\rangle=\omega\left(w^{\alpha}, w^{\beta}\right)=0 .
$$

We set $I_{j}=\left(I_{j}^{1}, \ldots, I_{j}^{k}\right)$.

In what follows we shall need several nondegeneracy conditions.

Condition A. The symmetric matrix

$$
K_{i}=\left(k_{i}^{\alpha \beta}\right), \quad k_{i}^{\alpha \beta}=\left\langle B_{i} w_{i}^{\alpha}, w_{i+1}^{\beta}\right\rangle,
$$

is nondegenerate for all $i$. We set $\left(k_{\alpha \beta i}\right)=\left(k_{i}^{\alpha \beta}\right)^{-1}=K_{i}^{-1}$.

Condition B. The matrix

$$
\bar{K}=\sum_{i=1}^{n} K_{i}^{-1}=\left(\bar{k}_{\alpha \beta}\right), \quad \bar{k}_{\alpha \beta}=\sum_{i=1}^{n} k_{\alpha \beta i},
$$

is nondegenerate.

Notation.

$$
\begin{gathered}
F_{i}=\left\{w_{i}: \mathbf{w} \in \Gamma\right\}=\operatorname{span}\left(w_{i}^{1}, \ldots, w_{i}^{k}\right) \subset E_{i}, \\
\widetilde{G}_{i} u_{i}=G_{i} u_{i}-k_{\alpha \beta i}\left\langle B_{i} w_{i-1}^{\alpha}, u_{i}\right\rangle w_{i}^{\beta}, \quad u_{i} \in E_{i}, \\
\widehat{\widetilde{G}}\left(u_{i}\right)=\left(\widetilde{G} u_{i}, \widetilde{G} u_{i+1}\right) .
\end{gathered}
$$

The period map $\widehat{\widetilde{G}} \widetilde{P}$ corresponds to the reduced linear Lagrangian system $(\widetilde{E}, \widetilde{\Lambda})$ with $\widetilde{E}_{i}=E_{i} / F_{i}$, which is obtained from the system $(E, \Lambda)$ using the Routh order reduction. It follows from Condition $\mathrm{A}$ that $\operatorname{dim} F_{i}=k$, and therefore the reduced configuration space $\widetilde{E}_{i}=E_{i} / F_{i}$ is identified with

$$
E_{i}^{\perp}=\left\{u \in E_{i}:\left\langle B_{i-1} w_{i-1}^{\alpha}, u\right\rangle=0, \alpha=1, \ldots, k\right\}
$$


by the projection $\Pi_{i}: E_{i} \rightarrow E_{i}^{\perp}$ :

$$
\Pi_{i} u=u_{i}-k_{\alpha \beta i}\left\langle B_{i-1} w_{i-1}^{\alpha}, u\right\rangle w_{i}^{\beta} .
$$

We can represent any vector $u_{i} \in E_{i}$ by

$$
u_{i}=v_{i}+\lambda_{\beta i} w_{i}^{\beta}, \quad v_{i}=\Pi_{i} u_{i} \in E_{i}^{\perp}, \lambda_{\beta i}=k_{\alpha \beta i-1}\left\langle B_{i-1} w_{i-1}^{\alpha}, u\right\rangle .
$$

Lemma 3.3. Suppose that $\mathbf{u}, \mathbf{v}$ are such that $u_{i}-v_{i} \in F_{i}$ :

$$
u_{i}=v_{i}+\lambda_{\beta i} w_{i}^{\beta} .
$$

Then $I_{i}^{\alpha}\left(u_{i}, u_{i+1}\right)=c^{\alpha}$ for all $\alpha=1, \ldots, k$ and all $i$ if and only if

$$
\Delta \lambda_{\alpha i}=\lambda_{\alpha i+1}-\lambda_{\alpha i}=k_{\alpha \beta i}\left(c^{\beta}-I_{i}^{\beta}\left(v_{i}, v_{i+1}^{\beta}\right)\right) .
$$

Equation (3.6) follows from

$$
c^{\alpha}=I_{i}^{\alpha}\left(u_{i}, u_{i+1}\right)=I_{i}^{\alpha}\left(v_{i}, v_{i+1}\right)+k_{i}^{\alpha \beta} \Delta \lambda_{\beta i} .
$$

We now state Routh's theorem for DLSs.

Theorem 3.4. Let $\mathbf{u}=\left(u_{i}\right), u_{i} \in E_{i}$, be a trajectory of the system $(E, \Lambda)$ such that $I_{i}\left(u_{i}, u_{i+1}\right)=0$. Then $\mathbf{v}=\left(v_{i}\right), v_{i}=\Pi_{i} u_{i} \in E_{i}^{\perp}$, is a trajectory of the linear Lagrangian system $\left(E^{\perp}, \Lambda^{\perp}\right)$ with the Lagrangian

where

$$
\Lambda_{i}^{\perp}\left(v_{i}, v_{i+1}\right)=\frac{1}{2}\left\langle A_{i} v_{i}, v_{i}\right\rangle-\left\langle B_{i} v_{i}, v_{i+1}\right\rangle-\frac{1}{2}\left\langle C_{i} v_{i}, v_{i}\right\rangle,
$$

$$
\left\langle C_{i} v_{i}, v_{i}\right\rangle=k_{\alpha \beta i}\left\langle B_{i} v_{i}, w_{i+1}^{\alpha}\right\rangle\left\langle B_{i} v_{i}, w_{i+1}^{\beta}\right\rangle .
$$

Conversely, if $\mathbf{v}$ is a trajectory of the system $\left(E^{\perp}, \Lambda^{\perp}\right)$, then there exists a trajectory $\mathbf{u}$ of the system $(E, \Lambda)$ defined $\bmod \Gamma$ such that $I_{i}\left(u_{i}, u_{i+1}\right)=0$ and $\Pi \mathbf{u}=\mathbf{v}$.

Definition 3.5. The quadratic action functional

$$
h^{\perp}(\mathbf{v}, \mathbf{v})=h(\mathbf{v}, \mathbf{v})-\sum_{i=1}^{n} k_{\alpha \beta i}\left\langle B_{i} v_{i}, w_{i+1}^{\alpha}\right\rangle\left\langle B_{i} v_{i}, w_{i+1}^{\beta}\right\rangle, \quad \mathbf{v} \in E^{\perp},
$$

corresponds to the reduced system $\left(E^{\perp}, \Lambda^{\perp}\right)$.

In the case of a $\widetilde{G}$-periodic trajectory $\mathbf{v}$ of period $n$, we have

$$
h^{\perp}(\mathbf{v}, \mathbf{v})=\sum_{i=1}^{n}\left\langle A_{i}^{\perp} v_{i}-B_{i}^{\perp^{*}} v_{i+1}-B_{i-1}^{\perp} v_{i-1}, v_{i}\right\rangle,
$$

where

$$
B_{0}^{\perp}=\widetilde{G}^{*}\left(x_{1}\right) B_{n}^{\perp}, \quad v_{n+1}=\widetilde{G} v_{1}, \quad v_{0}=\widetilde{G}^{-1} v_{n}, \quad A_{i}^{\perp}=R_{i}\left(A_{i}-C_{i}\right), \quad B_{i}^{\perp}=R_{i+1} B_{i},
$$

and $R_{i}: E_{i}^{*} \rightarrow\left(E_{i}^{\perp}\right)^{*}$ is the restriction map. The reduced system of variational equations has the form

$$
A_{i}^{\perp} v_{i}-B_{i}^{\perp *} v_{i+1}-B_{i-1}^{\perp} v_{i-1}=0, \quad B_{0}^{\perp}=\widetilde{G}^{*}\left(x_{1}\right) B_{n}^{\perp} .
$$

The linear operator $\mathbf{H}^{\perp}: X^{\perp} \rightarrow X^{\perp^{*}}$, which is called the reduced Hessian, corresponds to the bilinear form $h^{\perp}$. We also set

$$
\beta^{\perp}=\left|\prod_{i=1}^{n} \operatorname{det} B_{i}^{\perp}\right|^{-1}, \quad \sigma^{\perp}=\operatorname{sign}\left(\prod_{i=1}^{\mathrm{n}} \operatorname{det} \mathrm{B}_{\mathrm{i}}^{\perp}\right)^{-1} .
$$

Then Hill's theorem applied to the reduced system yields the following.

Corollary 3.6. In the above notation,

$$
\operatorname{det}(\widetilde{P}-I)=\sigma^{\perp}(-1)^{m-k} \beta^{\perp} \operatorname{det} \mathbf{H}^{\perp} .
$$


In order to use (3.8) in stability problems we need to know $\sigma^{\perp}$ and the Morse index of the form $h^{\perp}$. However, the relation between the Morse indices of $h$ and $h^{\perp}$ is not obvious. This is because the trajectory $\mathbf{u}$ with $I_{i}\left(u_{i}, u_{i+1}\right)=0$, to which the $\widetilde{G}$-periodic sequence $\mathbf{v} \in X^{\perp}$ corresponds, will not be a $G$-periodic sequence in general. In the following sections we present a connection between the indices of $h$ and $h^{\perp}$.

Lemma 3.7. We have

$$
\operatorname{det} B^{\perp}=\frac{\operatorname{det} B_{i}}{\operatorname{det} K_{i}}
$$

A proof can be found in [1].

Corollary 3.8. We have

$$
\sigma^{\perp}=(-1)^{\sum \text { ind } B_{i}^{\perp}}=\sigma(-1)^{\sum \text { ind } K_{i}} .
$$

3.3. Degeneracy of $h$. Let $\Gamma \subset X$ denote the space of $G$-periodic trajectories corresponding to a space $V \subset \operatorname{Ker}\left(\widehat{G}^{-1} P-I\right)$. This space is the linear span of vectors $\mathbf{w}_{1}, \ldots, \mathbf{w}_{k} \in X$. Since $\mathbf{H w}=0$ for $\mathbf{w} \in \Gamma$, the bilinear form $h(\mathbf{u}, \mathbf{u})=\langle\mathbf{H u}, \mathbf{u}\rangle$ is degenerate and defines a bilinear form $\widehat{h}$ on $\widehat{X}=X / \Gamma$. In order to compare $h$ with $h^{\perp}$, we restrict $h$ to a level set of the integrals $I$. Let

$$
\begin{aligned}
& Y=\left\{\mathbf{u} \in X: I_{1}\left(u_{1}, u_{2}\right)=\cdots=I_{n}\left(u_{n}, G u_{1}\right)\right\}, \\
& Z=\left\{\mathbf{v} \in X: v_{i} \in F_{i}\right\}=\left\{\mathbf{v} \in X: v_{i}=\lambda_{\alpha i} w_{i}^{\alpha}\right\} .
\end{aligned}
$$

Assertion 3.9. The spaces $Y$ and $Z$ are $h$-orthogonal, that is, $h(\mathbf{u}, \mathbf{v})=0$ for all $\mathbf{u} \in Y$ and $\mathbf{v} \in Z$. Moreover, $Y$ is an h-orthogonal complement of $Z$ :

$$
Y=\{\mathbf{u} \in X: h(\mathbf{u}, \mathbf{v})=0 \text { for all } \mathbf{v} \in Z\} .
$$

The restriction of $h$ to $Z$ has the form

$$
h(\mathbf{v}, \mathbf{v})=\sum_{i=1}^{n} k_{i}^{\alpha \beta} \Delta \lambda_{\alpha i} \Delta \lambda_{\beta i}=\sum_{i=1}^{n}\left\langle K_{i} \Delta \lambda_{i}, \Delta \lambda_{i}\right\rangle, \quad v_{i}=\lambda_{\alpha i} w_{i}^{\alpha} .
$$

See the proof in [1].

We obtain a quadratic form on $Z$ :

$$
\left.h\right|_{Z}(\mathbf{v}, \mathbf{v})=\left\langle\mathbf{H}_{Z} \mathbf{v}, \mathbf{v}\right\rangle=\sum_{i}\left\langle K_{i} \Delta \lambda_{i}, \Delta \lambda_{i}\right\rangle=\langle\mathbf{K} \lambda, \lambda\rangle,
$$

where $\lambda_{i} \in \mathbb{R}^{k}, \mathbf{H}_{Z}: Z \rightarrow Z^{*}$, and $\mathbf{K}: \mathbb{R}^{k n} \rightarrow \mathbb{R}^{k n}$ is defined by

$$
(\mathbf{K} \lambda)_{i}=K_{i-1} \Delta \lambda_{i-1}-K_{i} \Delta \lambda_{i} .
$$

We have $\operatorname{Ker} \mathbf{K}=\left\{\lambda \in \mathbb{R}^{k n}: K_{1} \Delta \lambda_{1}=\cdots=K_{n} \Delta \lambda_{n}\right\}$. Consequently, $\Delta \lambda_{i}=K_{i}^{-1} c$. Since $\sum_{i=1}^{n} \Delta \lambda_{i}=0$ it follows that $\bar{K} c=0$, where $\bar{K}$ is the matrix (3.3).

We now impose the nondegeneracy Condition B: the matrix $\bar{K}$ is nondegenerate. Then $\operatorname{Ker} \mathbf{K}=\left\{\lambda \in \mathbb{R}^{n k}: \lambda_{1}=\cdots=\lambda_{n}\right\}$ and $\operatorname{Ker} \mathbf{H}_{Z}=\Gamma$. We set $\widehat{Z}=Z / \Gamma$.

Assertion 3.10. The form $\left.h\right|_{\widehat{Z}}$ is nondegenerate and

$$
\text { ind }\left.h\right|_{\widehat{Z}}=\sum_{i=1}^{n} \text { ind } K_{i}-\text { ind } \bar{K} \text {. }
$$

Assertion 3.11. We have $Y+Z=X$ and $Y \cap Z=\Gamma$.

This follows from a lemma, which we state without proof. 
Lemma 3.12. For any $\mathbf{v} \in X$ there exists $\mathbf{u}=\Phi \mathbf{v} \in Y$ that is unique modulo $\Gamma$ such that $\mathbf{u}-\mathbf{v} \in Z$. In more detail, $u_{i}=v_{i}+\lambda_{\beta i} w_{i}^{\beta}$, where the $\lambda$ satisfy (3.6) and

$$
c^{\alpha}=\kappa^{\alpha \beta} \sum_{i=1}^{n} k_{\beta \delta i} I_{i}^{\delta}\left(v_{i}, v_{i+1}\right), \quad\left(\kappa^{\alpha \beta}\right)=\left(\bar{k}_{\alpha \beta}\right)^{-1}=\bar{K}^{-1} .
$$

The map $\Phi: X \rightarrow \widehat{Y}=Y / \Gamma$ satisfies the equation $\Phi(Z)=0$ and $\left.\Phi\right|_{Y}$ is the identity $\bmod \Gamma$. Hence Assertion 3.11 follows.

We set $\widehat{X}=X / \Gamma$ and denote the corresponding canonical projection by $\pi_{\Gamma}: X \rightarrow$ $\widehat{X}$. Then there exists a linear map $\widehat{\Pi}: \widehat{X} \rightarrow X^{\perp}$ such that the following diagram is commutative:

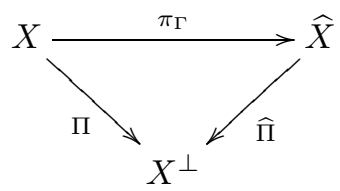

Corollary 3.13. The maps $\left.\widehat{\Pi}\right|_{\widehat{Y}}: \widehat{Y} \rightarrow X^{\perp}$ and $\left.\Phi\right|_{X^{\perp}}: X^{\perp} \rightarrow \widehat{Y}$ are mutually inverse isomorphisms.

Proof. If $\Pi \mathbf{v}=0$ for $\mathbf{v} \in Y$, then $\mathbf{v} \in Z$ and therefore $\mathbf{v} \in Y \cap Z=\Gamma$ by Assertion 3.11. It follows from the equation $Y+Z=X$ that $\left.\Pi\right|_{Y}$ is surjective. We also have $\Phi(Z)=0$.

The spaces $\widehat{Y}, \widehat{Z} \subset \widehat{X}$ are orthogonal with respect to the bilinear form $\widehat{h}$ on $\widehat{X}$. As we proved earlier, $\widehat{h}$ is nondegenerate on $\widehat{Z}$, and its index is given by equation (3.12).

From the viewpoint of Routh order reduction it is natural to consider the space

$$
Y^{0}=\left\{\mathbf{u} \in X: I_{i}\left(u_{i}, u_{i+1}\right)=0, i=1, \ldots, n, u_{n+1}=G u_{1}\right\} \subset Y .
$$

We define $\mathbf{d}_{\alpha}=\left(d_{\alpha i}\right) \in X^{*}$ by the equations

$$
d_{\alpha i}=k_{\alpha \beta i-1} B_{i-1} w_{i-1}^{\beta}-k_{\alpha \beta i} B_{i}^{*} w_{i+1}^{\beta}, \quad B_{0}=G\left(x_{1}\right)^{*} B_{n} .
$$

Note that $\left\langle d_{\alpha i}, w_{i}^{\alpha}\right\rangle=0$. We have

$$
\left\langle\mathbf{d}_{\alpha}, \mathbf{u}\right\rangle=\sum_{i=1}^{n} k_{\alpha \beta i} I_{i}^{\beta}\left(u_{i}, u_{i+1}\right), \quad \mathbf{u} \in X .
$$

Assertion 3.14. We have

$$
Y^{0}+Z=\left\{\mathbf{v} \in X:\left\langle\mathbf{d}_{\alpha}, \mathbf{v}\right\rangle=0, \alpha=1, \ldots, k\right\} .
$$

Proof. Let $\mathbf{v} \in X$. Then $\mathbf{v} \in Y^{0}+Z$ and $\mathbf{u}=\Phi(\mathbf{v}) \in Y^{0}$. By Lemma 3.12,

$$
0=\sum_{i=1}^{n} k_{\alpha \beta i} I_{i}^{\beta}\left(v_{i}, v_{i+1}\right)=\sum_{i=1}^{n}\left\langle d_{\alpha i}, v_{i}\right\rangle=\left\langle\mathbf{d}_{\alpha}, \mathbf{v}\right\rangle .
$$

Next we calculate the restriction $\left.h\right|_{Y}$. Let $h^{\perp}$ be the bilinear action form for the reduced system $\left(E^{\perp}, \Lambda^{\perp}\right)$ and let $h^{\top}=\left.h \circ \Phi\right|_{X^{\perp}}$, where $\Phi$ is the operator in Corollary 3.13, be the bilinear form on $X^{\perp}$ corresponding to $\left.h\right|_{Y}$. See the following commutative diagram:

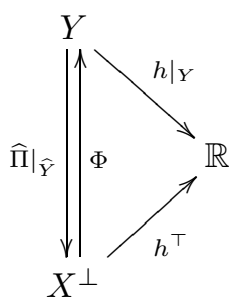


Assertion 3.15. For any $\mathbf{v} \in X^{\perp}$ we have

$$
h^{\top}(\mathbf{v}, \mathbf{v})=h^{\perp}(\mathbf{v}, \mathbf{v})+\bar{k}_{\alpha \beta} c^{\alpha} c^{\beta},
$$

where the coefficients $c^{\alpha}(\mathbf{v})$ are defined in (3.13).

3.4. The indices of $h$ and $h^{\perp}$. In this subsection we discuss the relation between ind $\left.h\right|_{Y}=\left.\operatorname{ind} \widehat{h}\right|_{\widehat{Y}}$ and ind $h^{\perp}$. By using the isomorphisms $\Phi: X^{\perp} \rightarrow \widehat{Y}$ and $\widehat{\Pi}: \widehat{Y} \rightarrow X^{\perp}$ we can restrict ourselves to comparing the indices of $h^{\perp}$ and $h^{\top}=h \circ \Phi$ on $X^{\perp}$. As we already mentioned, $h^{\top}$ and $h^{\perp}$ coincide on $X_{0}^{\perp}=\Pi Y^{0}$.

We shall need some assumptions on the structure of the eigenspace of 1 for the linear Poincaré map $\widehat{G}^{-1} P: W \rightarrow W$. Suppose that $V=\operatorname{Ker}\left(\widehat{G}^{-1} P-I\right)$ is isotropic. It is known that the generalized eigenspace $N=\operatorname{Ker}\left(\widehat{G}^{-1} P-I\right)^{2 m}$ is symplectic. Since $V \subset N$ is isotropic, $\operatorname{dim} N \geq 2 k$. We consider the least degenerate case $\operatorname{dim} N=2 k$. Then

$$
N=\operatorname{Ker}\left(\widehat{G}^{-1} P-I\right)^{2}=\left\{v \in W: \widehat{G}^{-1} P v-v \in V\right\}
$$

is symplectic and $V=\operatorname{Ker}\left(\widehat{G}^{-1} P-I\right)$ is a Lagrangian subspace of $N$. Consider the bilinear form

$$
b(v, w)=\omega\left(\left(\widehat{G}^{-1} P-I\right) v, w\right), \quad v, w \in N .
$$

A little later we shall show that $b$ is symmetric.

Let $\Sigma$ be the space of trajectories $\mathbf{v}$ of the variational equations corresponding to $v \in N$. Then $\Gamma \subset \Sigma$. The trajectories $\Sigma \backslash \Gamma$ are not $G$-periodic. The projection

$$
\Omega^{\perp}=\Pi \Sigma \subset X^{\perp}
$$

consists of $G$-periodic sequences. We have the natural map $\Psi=\Phi \Pi: \Sigma \rightarrow \widehat{Y}$. We set $\widehat{\Omega}=\Psi \Sigma$. We claim that $\Omega^{\perp}$ is orthogonal to $X_{0}^{\perp}=\Pi Y^{0}$ with respect to $h^{\perp}$ and $\widehat{\Omega}$ is orthogonal to $\widehat{Y}$ with respect to $h$. Since $h^{\top}$ and $h^{\perp}$ coincide on $X_{0}^{\perp}$, the difference of their indices is determined by their restrictions to the complement of $X_{0}^{\perp}$. Thus, if $\Omega^{\perp} \oplus X_{0}^{\perp}=X^{\perp}$, then the difference of the indices is determined by the forms $\left.h^{\top}\right|_{\Omega^{\perp}}$ and $\left.h^{\perp}\right|_{\Omega^{\perp}}$. Generally speaking, $X^{\perp} \neq \Omega^{\perp} \oplus X_{0}^{\perp}$. For this decomposition to hold we shall need one more nondegeneracy condition (Condition $\mathrm{C}$ below).

Consider the basis $w^{1}, \ldots, w^{k} \in V$ and the conjugate basis $q_{1}, \ldots, q_{k}$ in the skeworthogonal complement of $V$ in $N$. Then $w^{1}, \ldots, w^{k}, q_{1}, \ldots, q_{k}$ is a symplectic basis in $N$ and

$$
\begin{gathered}
\omega\left(w^{\alpha}, w^{\beta}\right)=0, \quad \omega\left(q_{\alpha}, q_{\beta}\right)=0, \\
\omega\left(w^{\alpha}, q_{\beta}\right)=\delta_{\beta}^{\alpha}, \quad \widehat{G}^{-1} P q_{\alpha}=q_{\alpha}+s_{\alpha \beta} w^{\beta} .
\end{gathered}
$$

In view of (3.17) and (3.18) we obtain

$$
b\left(q_{\alpha}, q_{\beta}\right)=s_{\alpha \delta} .
$$

Thus, the matrix $S=\left(s_{\alpha \delta}\right)$ is symmetric. We define symmetric matrices $A=\left(a_{\alpha \beta}\right)$ and $A^{\perp}=\left(a_{\alpha \beta}^{\perp}\right)$ as follows:

$$
a_{\alpha \beta}=s_{\alpha \delta} \kappa^{\delta \varepsilon} s_{\varepsilon \beta}-s_{\alpha \delta}, \quad a_{\alpha \beta}^{\perp}=s_{\alpha \beta}-\bar{k}_{\alpha \beta} .
$$

One more nondegeneracy condition is used in what follows.

Condition C. The matrix $A^{\perp}=\left(a_{\alpha \beta}^{\perp}\right)$ is nondegenerate.

Definition. We say that a periodic trajectory is nondegenerate $\bmod V$ if nondegeneracy Conditions A, B, C hold.

Theorem 3.16. Suppose that $\mathbf{x}$ is nondegenerate $\bmod V$. Then

$$
\text { ind } h-\text { ind }\left.h\right|_{Z}-\text { ind } h^{\perp}=\text { ind } A-\text { ind } A^{\perp} \text {. }
$$


Corollary 3.17. Suppose that $\mathbf{x}$ is nondegenerate $\bmod V$. Then

$$
\text { ind } h=\operatorname{ind} h^{\perp}+\text { ind }\left.h\right|_{Z}+\text { ind } b+\operatorname{ind} \bar{K} \bmod 2 \text {. }
$$

It follows from the equation $S A^{\perp}=A \bar{K}$ that

$$
\operatorname{sign} \operatorname{det} A=\operatorname{sign}\left(\operatorname{det} S \operatorname{det} K \operatorname{det} A^{\perp}\right) \text {. }
$$

Consequently, ind $A-$ ind $A^{\perp}=\operatorname{ind} b+\operatorname{ind} \bar{K} \bmod 2$.

Assertion 3.10 implies the following.

Corollary 3.18. We have ind $h=$ ind $h^{\perp}+\sum_{i=1}^{n}$ ind $K_{i}+\operatorname{ind} b \bmod 2$.

Equation (3.9) and Corollary 3.18 imply the following.

Corollary 3.19. We have $\sigma(-1)^{\text {ind } h}=\sigma^{\perp}(-1)^{\text {ind } h^{\perp}+\operatorname{ind} b}$.

\section{$\S 4$. HiLl'S FORMUla FOR $g$-PERIODIC TRAJECTORIES OF CONTINUOUS LAGRANGIAN SYSTEMS}

4.1. $g$-periodic trajectories of continuous Lagrangian systems. We consider a continuous Lagrangian system $(M, \mathscr{L})$ with configuration space $M^{m}$ and a smooth Lagrangian $\mathscr{L}(x, \dot{x}, t)$ on $T M \times \mathbb{R}$. Suppose that $\mathscr{L}$ is strictly convex with respect to the velocity $\dot{x} \in T_{x} M$; that is, $\mathscr{L}_{\dot{x} \dot{x}}$ is a positive-definite quadratic form on $T_{x} M$ for all $x \in M$. By definition, trajectories are solutions of the corresponding Lagrange equations.

Let $g: M \rightarrow M$ be a diffeomorphism.

Definition 4.1. A curve $\gamma: \mathbb{R} \rightarrow M$ is said to be $g$-periodic with period $\tau$ if $\gamma(t+\tau)=$ $g(\gamma(t))$ for any $t \in \mathbb{R}$. The class of all $g$-periodic curves on $M$ is denoted by $\Omega$.

We assume that the map $g$ preserves the Lagrangian in the following sense:

$$
\mathscr{L}(g(x), G(x) \dot{x}, t+\tau)=\mathscr{L}(x, \dot{x}, t) .
$$

Here, $G(x)$ is the differential of the map $g$ at point $x$.

Definition 4.2. The action functional $\mathscr{A}: \Omega \rightarrow \mathbb{R}$ is defined to be

$$
\mathscr{A}(\gamma) \stackrel{\text { def }}{=} \int_{I} \mathscr{L}(\gamma(t), \dot{\gamma}(t), t) d t
$$

where integration is conducted over any interval $I$ of length $\tau$.

Assertion 4.3. The action functional is well defined, since it is independent of the choice of $I$.

The proof is obvious.

Assertion 4.4. A curve $\gamma \in \Omega$ is a g-periodic trajectory if and only if $\gamma$ is a critical point of the action functional $\mathscr{A}$ on $\Omega$.

Proof. Let $\gamma(t, \alpha)=\gamma(t)+\alpha h(t)$ be the $g$-periodic variation of the curve $\gamma(t)$, where $h(t) \in T_{\gamma(t)} M$ is such that $h(t+\tau)=G(x) h(t)$. Then

$$
\begin{aligned}
& \delta \mathscr{A}(\gamma)=\left.\frac{d}{d \alpha}\right|_{\alpha=0} \int_{0}^{\tau} \mathscr{L}(\gamma+\alpha h, \dot{\gamma}+\alpha \dot{h}, t) d t \\
& =\int_{0}^{\tau}\left(\mathscr{L}_{x}(\gamma(t), \dot{\gamma}(t), t)-\frac{d}{d t} \mathscr{L}_{\dot{x}}(\gamma(t), \dot{\gamma}(t), t) \dot{h}(t)\right) h(t) d t+\left.\mathscr{L}_{\dot{x}}(\gamma(t), \dot{\gamma}(t), t) h(t)\right|_{0} ^{\tau} .
\end{aligned}
$$

The term $\left.\mathscr{L}_{\dot{x}} h\right|_{0} ^{\tau}$ is equal to zero, since condition (4.1) implies that

$$
\frac{\partial \mathscr{L}}{\partial \dot{x}}(g(x), G(x) \dot{x}, t+\tau) G(x)=\frac{\partial \mathscr{L}}{\partial \dot{x}}(x, \dot{x}, t) .
$$


4.2. The second variation of the action functional. The second variation of the action functional $\mathscr{A}(\gamma)$ at a point $\gamma \in \Omega$ is a symmetric bilinear form $h(\xi, \eta)$ on the space $X$ of $G$-periodic vector fields $\xi(t) \in E_{t}=T_{\gamma(t)} M$ along $\gamma$ :

$$
\begin{aligned}
& h(\xi, \xi)=\left.\frac{d^{2}}{d \alpha^{2}}\right|_{\alpha=0} \mathscr{A}\left(\gamma_{\alpha}\right)=\left.\frac{d^{2}}{d \alpha^{2}}\right|_{\alpha=0} \int_{0}^{\tau} \mathscr{L}(\gamma+\alpha \xi, \dot{\gamma}+\alpha \dot{\xi}, t) d t \\
&=\left.\frac{d}{d \alpha}\right|_{\alpha=0} \int_{0}^{\tau}\left(\mathscr{L}_{x} \xi(t)+\mathscr{L}_{\dot{x}} \dot{\xi}(t)\right) d t=\int_{0}^{\tau}\left(\left\langle\mathscr{L}_{x x}(\gamma, \dot{\gamma}, t) \xi(t), \xi(t)\right\rangle\right. \\
&\left.\quad+2\left\langle\mathscr{L}_{x \dot{x}}(\gamma, \dot{\gamma}, t) \xi(t), \dot{\xi}(t)\right\rangle+\left\langle\mathscr{L}_{\dot{x} \dot{x}}(\gamma, \dot{\gamma}, t) \dot{\xi}(t), \dot{\xi}(t)\right\rangle\right) d t .
\end{aligned}
$$

Definition 4.5. We define an inner product on $X$ :

$$
(v, w) \stackrel{\text { def }}{=}\langle B(t) v, w\rangle, \quad B(t) \stackrel{\text { def }}{=} \mathscr{L}_{\dot{x} \dot{x}}(\gamma, \dot{\gamma}, t),
$$

where $\langle\cdot, \cdot\rangle$ is the Euclidean inner product.

Assertion 4.6. The form $h$ can be uniquely represented as

$$
h(\xi, \eta)=\int_{0}^{\tau}(D \xi, D \eta)+(U \xi, \eta) d t
$$

where $U(t)=U^{*}(t)$ is a symmetric operator $U: X \rightarrow X$ and $D$ is a covariant derivative compatible with the metric; that is, it is a linear differential operator $D: X \rightarrow X$ such that

where $f: \mathbb{R} \rightarrow \mathbb{R}$ is an arbitrary scalar function.

$$
\frac{d}{d t}(\xi(t), \eta(t))=(D \xi(t), \eta(t))+(\xi(t), D \eta(t)), \quad \frac{d}{d t}(f \xi)=\dot{f} \xi+f \dot{\xi},
$$

A proof can be found in [1].

Corollary 4.7. The operators $D$ and $U$ preserve $G$-periodicity.

Corollary 4.8. The operator $D: X \rightarrow X$ is skew-symmetric with respect to the $L^{2}$-inner product

that is,

$$
(\xi(t), \eta(t))_{2}=\int_{0}^{\tau}(\xi(t), \eta(t)) d t
$$

$$
D(\xi(t), \eta(t))_{2}=-(\xi(t), D \eta(t))_{2} .
$$

Proof. For any pair of $G$-periodic vector fields $\xi, \eta$,

$$
\begin{aligned}
(D \xi, \eta)_{2} & =\int_{0}^{\tau}(D \xi(t), \eta(t)) d t=\int_{0}^{\tau}\left(\frac{d}{d t}(\xi(t), \eta(t))-(\xi(t), D \eta(t))\right) d t \\
& =\left.(\xi(t), \eta(t))\right|_{0} ^{\tau}-(\xi(t), D \eta(t))_{2}=-(\xi(t), D \eta(t))_{2}, \\
\left.(\xi, \eta)\right|_{t=\tau} & =\left.\left\langle\mathscr{L}_{\dot{x} \dot{x}} \xi, \eta\right\rangle\right|_{t=\tau}=\left.\left\langle\left(G^{T}\right)^{-1} \mathscr{L}_{\dot{x} \dot{x}} G^{-1} G \xi, G \eta\right\rangle\right|_{t=0} \\
& =\left.\left(G \xi,\left(G^{T}\right)^{-1} \mathscr{L}_{\dot{x} \dot{x}} G^{-1} G \eta\right)\right|_{t=0}=\left.(\xi, \eta)\right|_{t=0} .
\end{aligned}
$$

Corollary 4.9. For any $t \in \mathbb{R}$,

$$
(\xi(t+\tau), \eta(t+\tau))=(\xi(t), \eta(t))
$$

Corollary 4.10. The form $h$ satisfies $h(\xi, \eta)=\left(\left(-D^{2}+U\right) \xi, \eta\right)_{2} \stackrel{\text { def }}{=}(\mathbf{H} \xi, \eta)_{2}$, where $\mathbf{H} \stackrel{\text { def }}{=}-D^{2}+U$ is the Hessian of the action functional $\mathscr{A}$ with respect to the $L^{2}$-metric.

Definition 4.11. The equation $D^{2} \xi(t)=U(t) \xi(t)$ is called the variational equation of a $g$-periodic trajectory. 
From here on, we will be looking at the linearization $(E, \Lambda)$ of the Lagrangian system $(M, \mathscr{L})$ near a $g$-periodic trajectory, where $E=\left\{E_{t}\right\}, E_{t}=T_{\gamma(t)} M$, is a smooth family of vector spaces and $\Lambda(\xi, D \xi)=\frac{1}{2}(D \xi, D \xi)+\frac{1}{2}(U \xi, \xi)$ is a quadratic Lagrangian.

Definition 4.12. The linear period map is defined to be the map

$$
\begin{gathered}
P: T_{\dot{\gamma}(0)} T M \rightarrow T_{\dot{\gamma}(\tau)} T M, \\
P(\xi(0), D \xi(0))=(\xi(\tau), D \xi(\tau)) .
\end{gathered}
$$

We set

$$
\widehat{G}(x) \stackrel{\text { def }}{=}(d g(x)[], d g(x)[]) .
$$

The monodromy operator of the system of variational equations is defined to be

$$
\widehat{G}^{-1} \circ P: T_{\dot{\gamma}(0)} T M \rightarrow T_{\dot{\gamma}(0)} T M .
$$

Definition 4.13. Let

$$
\langle\langle\xi(t), \eta(t)\rangle\rangle \stackrel{\text { def }}{=}(D \xi(t), D \eta(t))_{2}+(\xi(t), \eta(t))_{2} \stackrel{\text { def }}{=}(\mathbf{B} \xi, \eta)_{2},
$$

where $\mathbf{B} \stackrel{\text { def }}{=}-D^{2}+I$ and $\left\langle\langle\rangle\right.$ is an inner product on the Hilbert space $X=T_{\gamma(t)} M$. Then

$$
h(\xi, \bar{\eta})=\left\langle\left\langle\mathbf{B}^{-1} \mathbf{H} \xi, \bar{\eta}\right\rangle\right\rangle=\langle\langle H \xi, \bar{\eta}\rangle,
$$

where $H \stackrel{\text { def }}{=} \mathbf{B}^{-1} \mathbf{H}$ is the Hessian of the action with respect to the inner product $\langle\langle\rangle$.

Remark 4.14. One can show that $H$ is a selfadjoint operator; that is,

$$
\langle\langle H \xi, \eta\rangle=\langle\langle\xi, H \eta\rangle \quad \text { for all } \xi(t), \eta(t) \in X .
$$

Definition 4.15. Let $Q: T_{\gamma(0)} M \rightarrow T_{\gamma(\tau)} M$ be the linearization of the map of parallel transport; that is, $Q \eta(0)=\eta(\tau)$, where $D \eta(t)=0$.

Assertion 4.16. The map $G^{-1} Q: T_{\gamma(0)} M \rightarrow T_{\gamma(0)} M$ is an orthogonal operator.

Proof. Since $D$ is a covariant derivative compatible with the metric, we have

$$
\frac{d}{d t}(\xi(t), \eta(t))=(D \xi(t), \eta(t))+(\xi(t), D \eta(t))=0,
$$

where $\xi(t)$ and $\eta(t)$ are vector fields that are parallel along $\gamma$.

Then

$$
(\xi(0), \eta(0))=(\xi(\tau), \eta(\tau))=(Q \xi(0), Q \eta(0)) .
$$

Since $(\xi, \eta)=(G \xi, G \eta)$, it follows that $G^{-1} Q$ preserves length. Consequently, $G^{-1} Q$ is an orthogonal operator.

Corollary 4.17. Roots of the polynomial $\operatorname{det}(Q-\rho G)$ have the form $\rho=e^{i \nu}, \nu \in \mathbb{R}$.

4.3. The generalized Hessian of the action. Let $\rho \in S^{1}$ and let $X_{\rho}$ be the space of complex $g$-quasiperiodic vector fields $\xi(t) \in E$, that is, such that $\xi(t+\tau)=\rho G \xi(t)$.

Definition 4.18. We call

$$
h(\xi, \eta)=\int_{0}^{\tau}((D \xi(t), D \bar{\eta}(t))+(U(t) \xi(t), \bar{\eta}(t))) d t
$$

the Hermitian $\rho$-index form. Let $X=X^{\mathbb{C}}$ be the space of complex $g$-periodic vector fields along $\gamma$. 
We identify $x$ and $x_{\rho}$ by the formula

$$
\xi(t) \in X \longmapsto e^{\mu t} \xi(t),
$$

where $\mu=\tau^{-1} \ln \rho$ and $0 \leq \operatorname{Im} \ln \rho<2 \pi$.

As a result we obtain a Hermitian form on $X$ :

$$
\begin{aligned}
h_{\rho}(\xi, \bar{\eta}) \stackrel{\text { def }}{=} & h\left(e^{\mu t} \xi, \overline{e^{\mu t} \eta}\right)=\left(D e^{\mu t} \xi, D \overline{e^{\mu t} \eta}\right)_{2}+\left(U e^{\mu t} \xi, \overline{e^{\mu t} \eta}\right)_{2} \\
= & \left(\mu e^{\mu t} \xi, \overline{D \bar{e}^{\mu t} \eta}\right)_{2}+\left(\mu e^{\mu t} \xi, \overline{D \overline{e^{\mu t} \eta}}\right)_{2}+\left(U e \mu t \xi, \overline{e^{\mu t} \eta}\right)_{2} \\
= & \left(\mu e^{\mu t} \xi, \bar{\mu} \overline{e^{\mu t} \eta}\right)_{2}+\left(\mu e^{\mu t} \xi, \overline{e^{\mu t}} D \eta\right)_{2}+\left(e^{\mu t} D \xi, \bar{\mu} \overline{e^{\mu t} \eta}\right)_{2} \\
& +\left(e^{\mu t} D \xi, \overline{e^{\mu t}} D \bar{\eta}\right)_{2}+\left(U e^{\mu t} \xi, \overline{e^{\mu t} \eta}\right)_{2} \\
= & (\xi, \bar{\eta})_{2}+(\mu \xi, D \bar{\eta})_{2}+(D \xi, \bar{\mu} \bar{\eta})_{2}+(D \xi, D \bar{\eta})_{2}+\left(U e^{\mu t}, \overline{e^{\mu t} \eta}\right)_{2} \\
= & ((D+\mu I) \xi, \overline{(D+\mu I) \eta})_{2}+(U \xi, \bar{\eta})_{2} \\
= & -\left((D+\mu I)^{2} \xi, \bar{\eta}\right)_{2}+(U \xi, \bar{\eta})_{2} \stackrel{\text { def }}{=}\left(\mathbf{H}_{\rho} \xi, \eta\right)_{2},
\end{aligned}
$$

where $\mathbf{H}_{\rho}=-(D+\mu I)^{2}+U$. We have taken into account that $\bar{\mu}=-\mu$ and $D$ is a real antisymmetric operator.

Definition 4.19. We define the $\rho$-Hessian

$$
\begin{gathered}
H_{\rho} \stackrel{\text { def }}{=} \mathbf{B}^{-1} \mathbf{H}_{\rho}=(-D+I)^{-1}\left(-(D+\mu I)^{2}+U\right), \\
H_{\rho}: X \rightarrow X, \quad h_{\rho}(\xi, \bar{\eta})=\left\langle\left\langle H_{\rho} \xi, \bar{\eta}\right\rangle\right\rangle .
\end{gathered}
$$

From now on we shall assume that $\rho \in \mathbb{C} \backslash\{0\}$.

Definition 4.20. Let $P_{N}: X \rightarrow X^{(N)}$ be the orthogonal projection onto the finitedimensional eigensubspace $X^{(N)}$ of the operator $D$ corresponding to eigenvalues in the set

$$
\Lambda_{N}=\{\nu \in \Lambda=\sigma(D):|\nu| \leq N\}
$$

Then let

$$
\operatorname{det} H_{\rho} \stackrel{\text { def }}{=} \lim _{N \rightarrow \infty} H_{\rho}^{(N)}, \quad H_{\rho}^{(N)}=P_{N} H_{\rho} P_{N}^{*}: X^{(N)} \rightarrow X^{(N)} .
$$

4.4. The spectrum of the operator $D$. The operator $D: X \rightarrow X$ is a real skewHermitian operator, that is, $D=\bar{D}=-D^{*}$; therefore $\sigma(D) \subset i \mathbb{R}$.

Assertion 4.21. The spectrum $\Lambda=\sigma(D) \subset i \mathbb{R}$ of the operator $D$ coincides with the set $\Lambda=\left\{\mu: \operatorname{det}\left(Q-e^{\mu \tau} G\right)=0\right\}$, where $G=d g(\gamma(0))$.

Proof. Let $(D+\mu I) \eta(t)=0$. Then $D\left(e^{\mu t} \eta(t)\right)=\mu e^{\mu t} \eta(t)+e^{\mu t} D \eta(t)=0$. Since $\eta(\tau)=$ $G \eta(0)$, we have $Q \eta(0)=e^{\mu \tau} \eta(\tau)=e^{\mu \tau} G \eta(0)$. Consequently, $\operatorname{det}\left(Q-e^{\mu \tau} G\right)=0$.

Corollary 4.22. Since $G^{-1} Q$ is an orthogonal operator, $-\mu \in \Lambda$ for any $\mu \in \Lambda$, and also $\mu+\frac{2 \pi i}{\tau} k \in \Lambda, k \in \mathbb{Z}$, that is, $\Lambda=\left\{\mu_{j}+\omega \mathbb{Z}\right\}$, where $\rho_{j}=e^{\mu_{j} \tau}$, and $\rho_{1}, \ldots, \rho_{m}$ are the roots of the equation $\operatorname{det}(Q-\rho G)=0,0 \leq \operatorname{Im} \mu_{\mathrm{j}}<2 \pi / \tau$.

Suppose that $\mu \notin \Lambda$. Then $H_{\rho}$ can be represented in the form

$$
H_{\rho}=-\left(-D^{2}+I\right)(D+\mu I)^{2}\left(I-(D+\mu I)^{-2} U\right)=S T,
$$

where $S=-\left(-D^{2}+I\right)(D+\mu I)^{2}$ and $T=\left(I-(D+\mu I)^{-2} U\right)$. Since $P_{N}^{*} P_{N}=I$, we have

$$
\operatorname{det} H_{\rho}=\lim _{N \rightarrow \infty} \operatorname{det}\left(P_{N} H_{\rho} P_{N}^{*}\right)=\lim _{N \rightarrow \infty} \operatorname{det}\left(P_{N} S P_{N}^{*} P_{N} T P_{N}^{*}\right)=\operatorname{det} S \operatorname{det} T .
$$


The form of the spectrum of the operator $D$ means that the finite-dimensional approximation of the determinant

$$
\operatorname{det} T(\mu) \stackrel{\text { def }}{=} f(\mu)=\lim _{N \rightarrow \infty} \operatorname{det}\left(P_{N} T P_{N}^{*}\right)
$$

converges absolutely for $\mu \notin \Lambda$, since $\operatorname{tr}\left((D+\mu I)^{-2} U\right)<\infty$. Therefore, $f$ is a holomorphic function on $\mathbb{C} \backslash \Lambda$ with poles at the points of the set $\Lambda$ which have multiplicity at most twice the multiplicity of the corresponding point of the spectrum of $D$.

Assertion 4.23. The function $f$ is periodic: $f(\mu+\omega)=f(\mu)$, where $\omega=2 \pi i / \tau$.

Proof. If $\xi \in X^{\mathbb{C}}$, then $e^{\omega t} \xi \in X^{\mathbb{C}}$. We have

$$
(D+\mu I)^{-2} e^{\omega t} \xi=e^{\omega t}(D+(\mu+\omega) I)^{2} \xi
$$

therefore,

$$
(D+\mu I)^{-2}\left(e^{\omega t}(D+(\mu+\omega) I)^{2} \xi\right)=e^{\omega t} \xi
$$

Then

$$
\left(I-(D+\mu I)^{-2} U\right) e^{\omega t} \xi=e^{\omega t}\left(I-(D+(\mu+\omega) I)^{-2} U\right) \xi .
$$

Consequently, the operators $T(\mu)$ and $T(\mu+\omega)$ are similar, that is, there exists an operator $A: X \rightarrow X$ such that $T(\mu)=A^{-1} T(\mu+\omega) A$.

Then $f(\mu)=\Psi\left(e^{\mu \tau}\right)$, where $\Psi(\rho)$ is a meromorphic function that has poles at the roots of the polynomial $\operatorname{det}(\rho G-Q)$. The multiplicity of each pole is at most twice the multiplicity of the corresponding root. Consequently, there exists a polynomial $g(\rho)$ of degree $\leq 2 m-1$ such that the functions $\Psi(\rho)$ and $g(\rho) \operatorname{det}^{-2}(\rho G-Q)$ have the same principal parts in the expansion into a Laurent series at each pole. Since $\Psi(\rho) \rightarrow 1$ as $\rho \rightarrow \infty$, by Liouville's theorem we have

$$
\Psi(\rho)-g(\rho) \operatorname{det}^{-2}(\rho G-Q)=\text { const }=1,
$$

and so

$$
\Psi(\rho)=g(\rho) \operatorname{det}^{-2}(\rho G-Q)+1 .
$$

We expand the determinant $\operatorname{det} S$ :

$$
\begin{aligned}
\operatorname{det} S & =\operatorname{det}\left(-\left(-D^{2}+I\right)^{-1}(D+\mu I)^{2}\right)=\lim _{N \rightarrow \infty} \prod_{\nu \in \Lambda_{N}} \frac{(\nu+\mu)^{2}}{\nu^{2}-1} \\
& =\lim _{N \rightarrow \infty}\left(-\mu^{2}\right)^{k} \prod_{\nu \in \Lambda, i \nu>0}\left(\frac{\nu^{2}-\mu^{2}}{\nu^{2}-1}\right)^{2}=(-1)^{k} \lim _{N \rightarrow \infty} \prod_{\nu \in \Lambda} \frac{\nu^{2}-\mu^{2}}{\nu^{2}-1},
\end{aligned}
$$

where $k$ is the multiplicity of zero in the spectrum of the operator $D$. We have used the fact that $\Lambda_{N}=-\Lambda_{N}$. Since $\Lambda=\left\{\nu_{i}+\omega \mathbb{Z}, i=1, \ldots, m\right\}$, the product

$$
\lim _{N \rightarrow \infty} \prod_{\nu \in \Lambda_{N}}\left(1-\frac{1+\mu^{2}}{\nu^{2}-1}\right)
$$

converges absolutely. Consequently, this product is a holomorphic function of the variables $\rho_{1}, \ldots, \rho_{m}, \rho$ for $\rho_{j} \neq e^{ \pm \tau}, j=1, \ldots, m$, and $\rho \neq 0$. Suppose that $\rho_{j} \neq 1$ and $\rho_{i} \neq \rho_{j}$ for $i \neq j$. Then $\nu_{i} \notin \omega \mathbb{Z}$ and $\nu_{i}-\nu_{j} \notin \omega \mathbb{Z}$ for $i \neq j$. We use Euler's formula to calculate the last product:

$$
\prod_{n \in \mathbb{Z}}\left(1-\frac{\mu^{2}}{(\nu+\omega n)^{2}}\right)=\frac{\cosh \mu \tau-\cosh \nu \tau}{1-\cosh \nu \tau}, \quad \nu \notin \omega \mathbb{Z} .
$$


We obtain

$$
\begin{aligned}
\prod_{\nu \in \Lambda} \frac{\nu^{2}-\mu^{2}}{\nu^{2}-1} & =\prod_{\nu \in \Lambda}\left(1-\frac{\mu^{2}}{\nu^{2}}\right)\left(1-\frac{1}{\nu^{2}}\right)^{-1} \\
& =\prod_{j=1}^{m} \prod_{n \in \mathbb{Z}}\left(1-\frac{\mu^{2}}{\left(\nu_{j}+\omega n\right)^{2}}\right)\left(1-\frac{1}{\left(\nu_{j}+\omega n\right)^{2}}\right)^{-1} \\
& =\prod_{j=1}^{m} \frac{\cosh \mu \tau-\cosh \nu_{j} \tau}{\cosh \tau-\cosh \nu_{j} \tau}=\prod_{j=1}^{m} \frac{\rho+\rho^{-1}-\rho_{j}-\rho_{j}^{-1}}{e^{\tau}+e^{-\tau}-\rho_{j}-\rho_{j}^{-1}} \\
& =\prod_{j=1}^{m} \frac{e^{\tau}\left(\rho-\rho_{j}\right)\left(\rho-\rho_{j}^{-1}\right)}{\rho\left(e^{\tau}-\rho_{j}\right)\left(e^{\tau}-\rho_{j}^{-1}\right)}=\prod_{j=1}^{m} \frac{e^{\tau}\left(\rho-\rho_{j}\right)^{2}}{\rho\left(e^{\tau}-\rho_{j}\right)^{2}}=\frac{e^{m \tau} \operatorname{det}^{2}(\rho G-Q)}{\rho^{m} \operatorname{det}^{2}\left(e^{\tau} G-Q\right)} .
\end{aligned}
$$

By continuity this holds for all $\rho_{1}, \ldots, \rho_{m} \neq e^{ \pm \tau}$ and $\rho \neq 0$. Then

$$
\rho^{m} \operatorname{det} H_{\rho}=(-1)^{k} \beta^{-1}\left(\operatorname{det}^{2}(\rho G-Q)+f(\rho)\right),
$$

where $\beta=e^{-m \tau} \operatorname{det}^{2}\left(e^{\tau} G-Q\right)$. Consequently, $F(\rho)=\rho^{m} \operatorname{det} H_{\rho}$ is a polynomial of degree $2 m$ with respect to $\rho$ with leading term $(-1)^{k} \beta^{-1} \operatorname{det}^{2} G$.

Assertion 4.24. The polynomials $F(\rho)$ and $E(\rho)=\operatorname{det}(P-\rho \widehat{G})$ have the same roots.

Proof. If $E(\rho)=0$, then there exists a nonzero $G$-periodic vector field $\xi(t) \in X$ such that

$$
\left(-D^{2}+U\right) e^{\mu \tau} \xi(t)=0 .
$$

Hence, $H_{\rho} \xi=0$. Suppose that $\rho \notin \sigma(Q)$, that is, $\mu \notin \Lambda$. Then $H_{\rho}=S T$, where $S$ is an invertible operator and $\operatorname{tr}(T-I)<\infty$. Therefore $T \xi=0$ implies $\operatorname{det} T=0$. Hence, $\operatorname{det} H_{\rho}=\operatorname{det} S \operatorname{det} T=0$, and so $G(\rho)=0$. If $\mu \in \Lambda$, then we repeat the same argument taking

$$
\begin{aligned}
& S=\left(-D^{2}+I\right)^{-1}\left(-(D+\mu I)^{2}+I\right), \\
& T=\left(-(D+\mu I)^{2}+I\right)^{-1}\left(-(D+\mu I)^{2}+U\right) .
\end{aligned}
$$

Conversely, suppose that $F(\rho)=0$. Then $\operatorname{det} H_{\rho}=0$ and so ker $H_{\rho} \neq 0$. One can show that the kernel ker $H_{\rho}$ consists of $g$-quasiperiodic vector fields. Hence, $\operatorname{det}(P-\rho \widehat{G})=0$, so that $E(\rho)=0$. Since the degrees of $E(\rho)$ and $F(\rho)$ are the same, they have common roots.

Assertion 4.25. We have $(-1)^{k}=\sigma(-1)^{m}$, where $\sigma=\operatorname{det}\left(G^{-1} Q\right)$.

Proof. Since $k$ is the multiplicity of zero in the spectrum of the operator $D$, it follows that $k$ is the dimension of the subspace of $T_{\gamma(0)} M$ on which the operator $G^{-1} Q$ is the identity, and $\sigma=(-1)^{n}$, where $n$ is the dimension of the subspace on which $G^{-1} Q$ is a reflection. Since the dimension of the complementary subspace is even and is equal to $m-k-n$, it follows that $(-1)^{k}=\sigma(-1)^{m}$.

Theorem 4.26 (Hill's formula). We have the formula

$$
\rho^{-m} \operatorname{det}(P-\rho \widehat{G})=\sigma(-1)^{m} \beta \operatorname{det} H_{\rho} .
$$

For $\rho=1$ we have $\operatorname{det}(P-\widehat{G})=\sigma(-1)^{m} \beta \operatorname{det} H$.

Corollary 4.27. The dynamical nondegeneracy of a g-periodic trajectory $\gamma$ is equivalent to its geometric nondegeneracy. 
4.5. Applications of Hill's formula to stability of $\boldsymbol{g}$-periodic trajectories. Let $\|\cdot\|$ be a $G$-invariant norm on $T M$. We shall only need the norm $\|\cdot\|$ in the spaces $T_{\gamma(t)} M$ so, for example, we can take the norm generated by the inner product $(\cdot, \cdot)$ in $T_{\gamma(t)} M$.

Definition 4.28. A $g$-periodic trajectory is said to be stable in the linear approximation if for any $\varepsilon>0$ there exists $\delta>0$ such that for any initial condition $v(0), D v(0) \in T_{\gamma(0)} M$ satisfying the conditions $\|v(0)\|<\delta,\|D v(0)\|<\delta$ we have

$$
\|v(t)\|<\varepsilon, \quad\|D v(t)\|<\varepsilon \quad \text { for all } t \in \mathbb{R}_{+},
$$

where $v(t)$ is a solution with the initial condition $(v(0), D v(0))$.

Remark 4.29. The fact that the norm $\|\cdot\|$ is $g$-invariant is important, since a $g$-periodic trajectory is not, in general, compact. This means that the choice of an arbitrary norm may transform an asymptotically stable trajectory into an unstable one, and vice versa.

Remark 4.30. Since $G$ preserves $\|\cdot\|$, we have

$$
\left\|G^{-n} v(t)\right\|=\|v(t)\|, \quad\left\|G^{-n} D v(t)\right\|=\|D v(t)\|, \quad t=n \tau+t_{0}, 0 \leq t_{0}<\tau .
$$

Assertion 4.31. The operators

$$
\left.\widehat{G}^{-1} P\right|_{t=0}: T_{\dot{\gamma}(0)} T M \rightarrow T_{\dot{\gamma}(0)} T M,\left.\quad \widehat{G}^{-1} P\right|_{t=\tau}: T_{\dot{\gamma}(0)} T M \rightarrow T_{\dot{\gamma}(0)} T M
$$

have the same eigenvalues: the equations

$$
\operatorname{det}(P(\gamma(0))-\rho \widehat{G}(\gamma(0)))=0 \quad \text { and } \quad \operatorname{det}(P(\gamma(\tau))-\rho \widehat{G}(\gamma(\tau)))=0
$$

are equivalent.

Proof. We consider the system of variational equations $(\tau \leq t<2 \tau)$ :

$$
\left\{\begin{array}{l}
D \xi(t)=v(t) \\
D v(t)=U(t) \xi(t), \quad \tau \leq t<2 \tau
\end{array}\right.
$$

or equivalently,

$$
\left\{\begin{array}{l}
D \xi(t+\tau)=v(t+\tau), \\
D v(t+\tau)=U(t+\tau) \xi(t+\tau), \quad 0 \leq t<\tau .
\end{array}\right.
$$

Since $\left.D\right|_{t+\tau} \xi(t+\tau)=\left.G D\right|_{t} G^{-1} \xi(t+\tau)$ and $U(t+\tau)=G U(t) G^{-1}$, we have

$$
\left\{\begin{array}{l}
\left.D\right|_{t} G^{-1} \xi(t+\tau)=G^{-1} v(t+\tau), \\
\left.D\right|_{t} G^{-1} v(t+\tau)=U(t) G^{-1} \xi(t+\tau), \quad 0 \leq t<\tau .
\end{array}\right.
$$

Then $\left.P\right|_{t=\tau} w=\widetilde{w}$ is equivalent to

$$
\left.P\right|_{t=0} \widehat{G}^{-1}(0) w=\widehat{G}^{-1}(0) \widetilde{w}, \quad w \in T_{\gamma(\tau)} M, \widetilde{w} \in T_{\gamma(2 \tau)} M .
$$

Consequently, $\left.\left(\widehat{G}^{-1} P\right)\right|_{t=\tau} w=\widetilde{w}$ is equivalent to

$$
\left.\left(\widehat{G}^{-1} P\right)\right|_{t=0} \widehat{G}^{-1}(0) w=\widehat{G}^{-1}(0) \widetilde{w}, \quad w, \widetilde{w} \in T_{\gamma(\tau)} M .
$$

Hence,

$$
\left(\widehat{G}^{-1} P\right)_{t=\tau}=\left.\widehat{G}(0)\left(\widehat{G}^{-1} P\right)\right|_{t=0} \widehat{G}^{-1}(0) .
$$

Assertion 4.32. We have

$$
\text { ind }\left.\gamma\right|_{[0, \tau]}=\text { ind }\left.\gamma\right|_{\left[t_{0}, t_{0}+\tau\right]} \text { for any } t_{0},
$$

where ind $\left.\gamma\right|_{\left[t_{0}, t_{0}+\tau\right]}$ is the Morse index of the curve $\gamma(t), t_{0} \leq t \leq \tau+t_{0}$. 
Proof. We need the case $t_{0}=\tau$; therefore we prove it. Let $\xi(t), \tau \leq t \leq 2 \tau$, be a vector field that belongs to the subspace on which the form $\left.h\right|_{[\tau, 2 \tau]}$ is negative-definite, that is,

$$
\left.h\right|_{[\tau, 2 \tau]}(\xi(t), \xi(t))=\int_{\tau}^{2 \tau}((D \xi, D \xi)+(U \xi, \xi)) d t<0 .
$$

Then

$$
\begin{aligned}
& \left.h\right|_{[\tau, 2 \tau]}(\xi(t), \xi(t)) \\
& =\int_{0}^{\tau}\left(\left(D G^{-1} \xi(t+\tau), D G^{-1} \xi(t+\tau)\right)+\left(U G^{-1} \xi(t+\tau), \xi(t+\tau)\right)\right) d t \\
& =\left.h\right|_{[0, \tau]}\left(G^{-1} \xi(t+\tau), \xi(t+\tau)\right)<0 .
\end{aligned}
$$

Since $G(\gamma(t))$ is an isomorphism between $\bigcup_{0 \leq t \leq \tau} T_{\gamma(t)} M$ and $\bigcup_{\tau \leq t \leq 2 \tau} T_{\gamma(t)} M$, the dimension of the negative subspace of $\left.h\right|_{[0, \tau]}(\bar{\xi}, \bar{\xi})$ coincides with the dimension of the negative subspace of $\left.h\right|_{[\tau, 2 \tau]}(\xi, \xi)$.

See 18 for more details about the Morse index.

Definition 4.33. The index of a $g$-periodic curve $\gamma$ is defined as the index of $\left.\gamma\right|_{t_{0} \leq t \leq t_{0}+\tau}$. It follows from Assertion 4.32 that it is well defined, that is, it is independent of the choice of $t_{0}$.

Assertion 4.34. Suppose that the Poincaré map $\widehat{G}^{-1} P$ has a multiplier greater than 1 in absolute value. Then the g-periodic trajectory is unstable in the linear approximation.

The proof is similar to the case of a periodic trajectory.

Assertion 4.35. Suppose that $\sigma(-1)^{m+\operatorname{ind} \gamma}<0$. Then the g-periodic trajectory is unstable.

Proof. $F(\rho)=\operatorname{det}(P-\rho \widehat{G})$ is a polynomial of degree $2 m$. Since $\operatorname{det} P>0$, we have $F(+\infty)=+\infty$. Since $F(1)<0$, there exists a real root $\rho$ greater than 1 .

Corollary 4.36. Suppose that $\sigma(-1)^{m}<0$ and let $\gamma$ be a nondegenerate local minimum of the action functional $\mathscr{A}$. Then the g-periodic trajectory $\gamma$ is unstable.

\section{§5. Degeneracy in Hill's formula}

In this section we consider the case where a $g$-periodic trajectory $\gamma$ is degenerate, that is, the system of variational equations has a nonzero $\widehat{G}$-periodic solution $\zeta(t)$. Then it follows from Hill's formula that the Poincaré map $\widehat{G}^{-1} P$ has a nonzero eigenvector $w: \widehat{G}^{-1} P w=w$. This happens, for example, when a nonlinear Lagrangian system $(\mathscr{M}, \mathscr{L})$ has a $g$-invariant first integral $\mathscr{J}$ that is nondegenerate on a trajectory $\gamma(t)$. Then one can show that the system of variational equations has a nonzero $\widehat{G}$-periodic solution.

5.1. A continuous symmetry group. We represent the system $(M, \mathscr{L})$ as a Hamiltonian system with Hamiltonian $\mathscr{H}$. Suppose that the system admits an algebra $\mathfrak{g}$ of Hamiltonian symmetry fields generated by Hamiltonians $\mathscr{J}_{\mathbf{v}}, \mathbf{v} \in \mathfrak{g}$. Suppose also that

$$
v(g x)=G v(x) \text { for all } \mathbf{v} \in \mathfrak{g} .
$$

Condition (5.1) holds, for example, when $g$ is an element of this symmetry group. Let $\gamma$ be a $g$-periodic solution in the phase space. For $\mathbf{v} \in \mathfrak{g}$, the vector field $\zeta(t)=\mathbf{v}(\gamma(t))$ is a $\widehat{G}$-periodic solution of the system of variational equations, and

$$
P w=\widehat{G} w, \quad w=\mathbf{v}(\gamma(0)) .
$$


If the system is autonomous, then the Hamiltonian vector field belongs to $\mathfrak{g}$ and the corresponding eigenvector is $w=\dot{\gamma}(0)$.

Following 1, we consider the case when $\mathfrak{g}$ is a $k$-dimensional commutative algebra. Then the corresponding eigensubspace $V \subset \operatorname{Ker}\left(\widehat{G}^{-1} P-I\right)$ is isotropic and the eigenvalue 1 has multiplicity at least $2 k$. We use Routh's method to eliminate the degeneracy. For further details on this see [16].

5.2. Routh's reduction for linear Lagrangian systems. Suppose that a $g$-periodic trajectory is degenerate, that is, the system of variational equations has a nonzero $G$ periodic solution $\zeta(t)$. In this case the linear Poincaré map $\widehat{G}^{-1} P$ has an eigenvector $w=\widehat{G}^{-1} P w$ corresponding to the eigenvalue 1 .

Assertion 5.1. The system of variational equations has the linear first integral $I_{\zeta}(\xi, D \xi)$ $=(\zeta, D \xi)-(\xi, D \zeta)$.

Proof. We have

$$
\frac{d}{d t} I_{\zeta}(\xi(t), D \xi(t))=\left(\zeta, D^{2} \xi\right)-\left(\xi, D^{2} \zeta\right)=(\zeta, U \xi)-(\xi, U \zeta)=0
$$

In fact, $I_{\zeta}(\xi, D \xi)=\omega(w, v)=J_{w}(v)$ is the value of the symplectic form on the vectors $v, w \in W$ corresponding to $\xi, \zeta$.

Suppose that the Poincaré map $\widehat{G}^{-1} P$ has several eigenvectors corresponding to the eigenvalue 1. Suppose that $V \subset \operatorname{Ker}\left(\widehat{G}^{-1} P-I\right)$ is an isotropic subspace, and let $\Gamma \subset X$ be the corresponding space of $\widehat{G}$-periodic solutions of the system $(E, \Lambda)$. Let $w^{1}, \ldots, w^{k}$ be a basis for $V$ and let $\zeta^{1}, \ldots, \zeta^{k} \in \Gamma$ be the corresponding independent solutions. The system $(E, \Lambda)$ has the first integrals

$$
I^{\alpha}(\xi, D \xi)=\left(\zeta^{\alpha}, D \xi\right)-\left(\xi, D \zeta^{\alpha}\right), \quad \alpha=1, \ldots, k .
$$

Since the corresponding space is isotropic these integrals commute:

$$
I^{\alpha}\left(\zeta^{\beta}, D \zeta^{\beta}\right)=\left(\zeta^{\alpha}, D \zeta^{\beta}\right)-\left(\zeta^{\beta}, D \zeta^{\alpha}\right)=\omega\left(w^{\alpha}, w^{\beta}\right)=0 .
$$

Notation. $I=\left(I^{1}, \ldots, I^{k}\right)$;

$$
F_{t}=\{\zeta(t): \zeta \in \Gamma\}=\operatorname{span}\left\{\zeta^{1}(t), \ldots, \zeta^{k}(t)\right\} .
$$

We adopt the following nondegeneracy assumption:

Assumption A. $\operatorname{dim} F_{t}=k$ for all $t$.

Equivalently, the vectors $\zeta^{1}(t), \ldots, \zeta^{k}(t) \in E_{t}$ are independent for all $t$. Then the Gram matrix

$$
K=\left(k^{\alpha \beta}\right), \quad k^{\alpha \beta}(t)=\left(\zeta^{\alpha}(t), \zeta^{\beta}(t)\right)
$$

is nondegenerate for all $t$.

Remark 5.2. One can show [1] that Assumption A is redundant; namely, the singular set $\Sigma=\left\{t \in \mathbb{R} / \tau \mathbb{Z}: \operatorname{dim} F_{t}<k\right\}$ is finite and the family $\left(F_{t}\right)_{t \notin \Sigma}$ can be extended to a

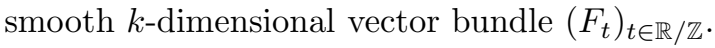

In Routh's reduction of the linear system $(E, \Lambda)$, the reduced configuration space $\widetilde{E}_{t}=E_{t} / F_{t}$ can be identified with

$$
E_{t}^{\perp}=\left\{u \in E_{t}:(u, w)=0 \quad \text { for all } w \in F_{t}\right\}
$$

by the orthogonal projection $\Pi=\Pi_{t}: E_{t} \rightarrow E_{t}^{\perp}$. For a smooth field $\xi(t) \in E_{t}$, we denote $D^{\perp} \xi(t)=\Pi_{t} D \xi(t)$. In explicit form:

$$
\Pi \xi=\xi-k_{\alpha \beta}\left(\xi, \zeta^{\beta}\right) \zeta^{\alpha}, \quad D^{\perp} \xi=D \xi-k_{\alpha \beta}\left(D \xi, \zeta^{\beta}\right) \zeta^{\alpha},
$$


where $K^{-1}=\left(k_{\alpha \beta}\right)$ is the inverse of the Gram matrix $K=\left(k^{\alpha \beta}\right)$.

We define the reduced Routh Lagrangian $\Lambda^{\perp}$ on $E^{\perp}=\left(E_{t}^{\perp}\right)$ by

$$
\Lambda^{\perp}(\eta, D \eta)=\frac{1}{2}\left(D^{\perp} \eta, D^{\perp} \eta\right)+\frac{1}{2}\left(U^{\perp} \eta, \eta\right), \quad \eta(t), D^{\perp} \eta(t) \in E_{t}^{\perp},
$$

where the symmetric operator $U^{\perp}(t): E_{t}^{\perp} \rightarrow E_{t}^{\perp}$ is defined by

$$
\left(U^{\perp} u, u\right)=(U u, u)-3 k_{\alpha \beta}\left(u, D^{\perp} \zeta^{\alpha}\right)\left(u, D^{\perp} \zeta^{\beta}\right), \quad u \in E_{t}^{\perp} .
$$

Hence, $U^{\perp}=\Pi U-3 C$, where 2

$$
C u=k_{\alpha \beta}\left(u, D^{\perp} \zeta^{\alpha}\right) D^{\perp} \zeta^{\beta} .
$$

The bilinear action form of the system $\left(E^{\perp}, \Lambda^{\perp}\right)$ has the form

$$
\frac{1}{2} h^{\perp}(\eta, \eta)=\int_{0}^{\tau} \Lambda^{\perp}\left(\eta, D^{\perp} \eta\right) d t, \quad \eta(t) \in E_{t}^{\perp} .
$$

Lemma 5.3. Let

$$
\eta(t)-\xi(t) \in F_{t}, \quad \xi(t)=\eta(t)+\lambda_{\alpha}(t) \zeta^{\alpha}(t) .
$$

Then $\xi$ satisfies the equation $I_{i}^{\alpha}(\xi, D \xi)=c^{\alpha}$ for all $\alpha=1, \ldots, k$ if and only if

$$
\dot{\lambda}_{\alpha}=k_{\alpha \beta}\left(c^{\beta}-I^{\beta}(\eta, D \eta)\right) \text {. }
$$

Indeed, $I^{\alpha}(\xi, D \xi)=I^{\alpha}(\eta, D \eta)+k^{\alpha \beta} \dot{\lambda}_{\beta}$.

Recall Routh's theorem for linear Lagrangian systems.

Theorem 5.4. Let $\xi(t) \in E_{t}$ be a solution of the system $(E, \Lambda)$ such that $I(\xi, D \xi) \equiv 0$. Then $\eta(t)=\Pi \xi(t) \in E_{t}^{\perp}$ is a solution of the system $\left(E^{\perp}, \Lambda^{\perp}\right)$. Conversely, if $\eta(t)$ is a solution of the system $\left(E^{\perp}, \Lambda^{\perp}\right)$, then there exists a solution $\xi(t)$ of the system $(E, \Lambda)$, that is unique $\bmod \Gamma$, such that $I(\xi, D \xi)=0$ and $\eta(t)=\Pi \xi(t)$.

The proof is similar to the proof in [1].

5.3. Hill's formula for the reduced Poincaré map. Let $G^{\perp}: E_{t} \rightarrow E_{t}^{\perp}$ denote the operator defined by the relation

$$
G^{\perp}(t) \xi(t)=G(t) \xi(t)-s_{\alpha \beta}\left(G \xi(t), G \zeta^{\alpha}\right) G \zeta^{\beta}, \quad \xi(t) \in E_{t} .
$$

In other words, $G^{\perp}(t)=\Pi_{t} G(t)$. We observe that for all $\xi(t) \in E_{t}^{\perp}, \zeta(t) \in \Gamma$ the following holds:

$$
(G \xi(t), \zeta(t+\tau))=(\xi(t), \zeta(t))=0 .
$$

Definition 5.5. Let $\widetilde{P}: E_{0}^{\perp} \oplus E_{0}^{\perp} \rightarrow E_{\tau}^{\perp} \oplus E_{\tau}^{\perp}$ be the period map over period $\tau$ of the system of variational equations

$$
\begin{gathered}
D^{\perp 2} \xi(t)=U^{\perp} \xi(t), \quad \widehat{P}=\left(\widehat{G}^{\perp}\right)^{-1} \widetilde{P}: E_{0}^{\perp} \oplus E_{0}^{\perp} \rightarrow E_{0}^{\perp} \oplus E_{0}^{\perp}, \\
\widehat{G}^{\perp}: \widetilde{W}_{0} \rightarrow \widetilde{W}_{\tau}, \quad \widehat{G}^{\perp}(\xi(t), \eta(t))=\left(G^{\perp} \xi(t), G^{\perp} \eta(t)\right) .
\end{gathered}
$$

Definition 5.6. We set $X^{\perp}=\left\{\eta \in X: \eta(t) \in E_{t}^{\perp}\right\}$ and denote the Hesse operator for the reduced system $\left(E^{\perp}, \Lambda^{\perp}\right)$ by

$$
H^{\perp}=\left(-D^{\perp 2}+I\right)^{-1}\left(-D^{\perp 2}+U^{\perp}\right): X^{\perp} \rightarrow X^{\perp} .
$$

Let $Q^{\perp}: E_{0}^{\perp} \rightarrow E_{0}^{\perp}$ be the linear parallel transport map corresponding to the equation $D^{\perp} \eta=0$. We set $\sigma^{\perp}=\operatorname{det} Q^{\perp}= \pm 1$. If Assumption A holds, then $\sigma=\sigma^{\perp}$, since the vector bundle $F$ is orientable.

We can now write Hill's formula for the reduced Poincaré map $\widetilde{P}: \widetilde{W} \rightarrow \widetilde{W}$.

${ }^{2} C$ is independent of the choice of basis. 
Theorem 5.7. We have

$$
\begin{aligned}
& \operatorname{det}(\widehat{P}-I)=\sigma^{\perp}(-1)^{m-k} \beta \operatorname{det} H^{\perp}, \\
& \beta=e^{(m-k) \tau} \operatorname{det}^{-2}\left(e^{\tau} G^{\perp}-Q^{\perp}\right)>0 .
\end{aligned}
$$

Note that $H^{\perp} \neq\left. H\right|_{X^{\perp}}$ in the general case, except when $D^{\perp} \zeta=0$. This is because the space $X^{\perp}$ of $G^{\perp}$-periodic $\eta(t) \in E_{t}^{\perp}$ does not correspond to the space of $G$-periodic $\xi(t) \in E_{t}$ such that $I(\xi, D \xi)=0$. Therefore $h^{\perp}$ is not the restriction of $h$ to $X^{\perp}$, as in the geodesic case. Therefore we need to discuss the connection between $h^{\perp}$ and $h$.

\subsection{Eliminating the degeneracy in the second variation.}

Definition 5.8. We define subspaces $Y, Z \subset X$ by the formulae

$$
\begin{aligned}
& Y=\{\xi \in X: I(\xi, D \xi) \equiv \text { const }\}, \\
& Z=\left\{\xi \in X: \xi(t) \in F_{t} \text { for all } t\right\} .
\end{aligned}
$$

If $\zeta^{1}, \ldots, \zeta^{k} \in \Gamma$ are a basic set of $G$-periodic solutions, then

$$
Z=\left\{\xi(t)=\lambda_{\alpha}(t) \zeta^{\alpha}(t): \lambda_{\alpha}(t+\tau)=\lambda_{\alpha}(t), \int_{0}^{\tau} k^{\alpha \beta} \dot{\lambda}_{\alpha} \dot{\lambda}_{\beta} d t<\infty\right\} .
$$

Lemma 5.9. For any $\eta \in X$ there exists $\xi=\Phi(\eta) \in Y$ that is unique $\bmod \Gamma$ such that $\xi-\eta \in Z$. In explicit form: $\eta=\xi-\lambda_{\alpha} \zeta^{\alpha}$, where $\lambda_{\alpha}$ satisfies (5.8) and $c^{\alpha}=c^{\alpha}(\eta)$ is given by

$$
c^{\alpha}=\kappa^{\alpha \beta} \int_{0}^{\tau} k_{\beta \delta} I^{\delta}(\eta, D \eta) d t, \quad\left(\kappa^{\alpha \beta}\right)=\left(\bar{k}_{\alpha \beta}\right)^{-1}, \quad \bar{k}_{\alpha \beta}=\int_{0}^{\tau} k_{\alpha \beta} d t .
$$

This follows from Lemma 5.3 for $G$-periodic $\xi, \eta$.

Thus, we have constructed a projection $\Phi: X \rightarrow \widehat{Y}=Y / \Gamma$ that is the identity on $Y$ and is equal to zero on $Z$. We obtain the following.

Assertion 5.10. We have $Y \cap Z=\Gamma$ and $Y+Z=X$.

Assertion 5.11. The spaces $Y, Z$ are h-orthogonal, that is, $h(\xi, \eta)=0$ for all $\xi \in Z$, $\eta \in Y$. The restriction of $h$ to $Z$ has the form

$$
h\left(\lambda_{\alpha} \zeta^{\alpha}, \lambda_{\beta} \zeta^{\beta}\right)=\int_{0}^{\tau} k^{\alpha \beta} \dot{\lambda}_{\alpha} \dot{\lambda}_{\beta} d t
$$

Proof. We take $\xi \in Z, \xi(t)=\lambda_{\alpha}(t) \zeta^{\alpha}(t)$. Then

$$
\begin{aligned}
h\left(\lambda_{\alpha} \zeta^{\alpha}, \eta\right) & =\int_{0}^{\tau}\left(\left(D\left(\lambda_{\alpha} \zeta^{\alpha}\right), D \eta\right)+\left(U \lambda_{\alpha} \zeta^{\alpha}, \eta\right)\right) d t \\
& =\int_{0}^{\tau}\left(\left(\dot{\lambda}_{\alpha} \zeta^{\alpha}, D \eta\right)+\left(\lambda_{\alpha} D \zeta^{\alpha}, D \eta\right)+\left(\lambda_{\alpha} D^{2} \zeta^{\alpha}, \eta\right)\right) d t \\
& =\int_{0}^{\tau}\left(\frac{d}{d t}\left(\eta, \lambda_{\alpha} D \zeta^{\alpha}\right)+\dot{\lambda}_{\alpha} I^{\alpha}(\eta, D \eta)\right) d t \\
& =\left.\left(\eta, \lambda_{\alpha} D \zeta^{\alpha}\right)\right|_{0} ^{\tau}+\int_{0}^{\tau} \dot{\lambda}_{\alpha} I^{\alpha}(\eta, D \eta) d t .
\end{aligned}
$$

Here we have used the fact that $\zeta$ satisfies the system of variational equations. If $\eta=$ $\lambda_{\alpha} \zeta^{\alpha}$, then $I^{\alpha}(\eta, D \eta)=k^{\alpha \beta} \dot{\lambda}_{\beta}$. Therefore,

$$
h\left(\lambda_{\alpha} \zeta^{\alpha}, \lambda_{\beta} \zeta^{\beta}\right)=\int_{0}^{\tau} k^{\alpha \beta} \dot{\lambda}_{\alpha} \dot{\lambda}_{\beta} d t+\left.\left(\lambda_{\alpha} \zeta^{\alpha}, \lambda_{\beta} D \zeta^{\beta}\right)\right|_{0} ^{\tau} .
$$

If $\eta \in Y$ is $G$-periodic and $\lambda_{\alpha}(t+\tau)=\lambda_{\alpha}(t)$, then (5.13) gives 0, while (5.14) gives (5.12). Assertion 5.11 is proved. 
Notation. Let $\widehat{X}=X / \Gamma, \widehat{Y}=Y / \Gamma, \widehat{Z}=Z / \Gamma$.

Then $\widehat{Y} \oplus \widehat{Z}=\widehat{X}$. The bilinear form $h$ defines a form $\widehat{h}$ on $\widehat{X}$ and $\widehat{Y} \perp_{\widehat{h}} \widehat{Z}$, while $\left.\widehat{h}\right|_{\widehat{Z}}$ is positive-definite. We obtain the following.

Corollary 5.12. We have ind $h=\left.\operatorname{ind} \widehat{h}\right|_{\widehat{Y}}$ and null $h=\left.\operatorname{null} \widehat{h}\right|_{\widehat{Y}}+k$.

Corollary 5.13. The projection $\Pi: X \rightarrow X^{\perp}$ defines an isomorphism

$$
\left.\widehat{\Pi}\right|_{\widehat{Y}}: \widehat{Y} \rightarrow X^{\perp}, \quad\left(\left.\Pi\right|_{\widehat{Y}}\right)^{-1}=\left.\Phi\right|_{X^{\perp}} .
$$

Indeed, if $\Pi \xi=0$ for $\xi \in Y$, then $\xi \in Z$, so that $\xi \in Y \cap Z=\Gamma$ (Assertion 5.10). Similarly, $Y+Z=X$ implies that $\Pi(Y)=X^{\perp}$.

We will now calculate the restriction $\left.h\right|_{Y}$. Let $h^{\perp}(\eta, \eta)$ be the bilinear form of the reduced system $\left(E^{\perp}, \Lambda^{\perp}\right)$.

Assertion 5.14. Let $h^{\top}=\left.h\right|_{Y} \circ \widehat{\Pi}^{-1}$ be the bilinear form on $X^{\perp}$ corresponding to the form $\left.h\right|_{Y}$. Then for every $\eta \in X^{\perp}$ we have

$$
h^{\top}(\eta, \eta)=h^{\perp}(\eta, \eta)+\bar{k}_{\alpha \beta} c^{\alpha} c^{\beta},
$$

where $c^{\alpha}(\eta)=I^{\alpha}(\xi, D \xi), \xi=\Phi \eta$, are defined by formula (5.11).

This is a consequence of the following more general formula.

Lemma 5.15. Let $\xi(t)=\eta(t)+\lambda_{\alpha}(t) \zeta^{\alpha}(t), 0 \leq t \leq \tau$, be the same as in Lemma 5.3 . Then

$$
\begin{aligned}
h(\xi, \xi)=h(\eta, \eta)-\int_{0}^{\tau} k_{\alpha \beta}\left(I^{\alpha}(\right. & \left., D \eta)-c^{\alpha}\right)\left(I^{\beta}(\eta, D \eta)-c^{\beta}\right) d t \\
& +\left.\left(\left(\lambda_{\alpha} \zeta^{\alpha}, \lambda_{\beta} D \zeta^{\beta}\right)+2\left(\lambda_{\alpha} \eta, D \zeta^{\alpha}\right)+2 c^{\alpha} \lambda_{\alpha}\right)\right|_{0} ^{\tau}
\end{aligned}
$$

Proof. We have

$$
h(\xi, \xi)=h(\eta, \eta)+2 h\left(\eta, \lambda_{\alpha} \zeta^{\alpha}\right)+h\left(\lambda_{\alpha} \zeta^{\alpha}, \lambda_{\beta} \zeta^{\beta}\right) .
$$

Now (5.17) follows from (5.13)-(5.14) and (5.8).

Proof of Assertion 5.14. If $\eta$ is a $G$-periodic vector field and the functions $\lambda_{\alpha}$ are periodic, then

$$
\left(\lambda_{\alpha} \zeta^{\alpha}, \lambda_{\beta} D \zeta^{\beta}\right)+2\left(\lambda_{\alpha} \eta, D \zeta^{\alpha}\right)+\left.2 c^{\alpha} \lambda_{\alpha}\right|_{0} ^{\tau}=0
$$

By (5.11),

$$
\begin{aligned}
\int_{0}^{\tau} & k_{\alpha \beta}\left(c^{\alpha}-I^{\alpha}(\eta, D \eta)\right)\left(c^{\beta}-I^{\beta}(\eta, D \eta)\right) d t \\
\quad= & \int_{0}^{\tau} k_{\alpha \beta} I^{\alpha}(\eta, D \eta) I^{\beta}(\eta, D \eta) d t-2 \int_{0}^{\tau} k_{\alpha \beta} c^{\alpha} I^{\beta}(\eta, D \eta) d t+\bar{k}_{\alpha \beta} c^{\alpha} c^{\beta} \\
& =\int_{0}^{\tau} k_{\alpha \beta} I^{\alpha}(\eta, D \eta) I^{\beta}(\eta, D \eta) d t-\bar{k}_{\alpha \beta} c^{\alpha} c^{\beta}
\end{aligned}
$$

We now use the fact that $\eta=\Pi \xi \in X^{\perp}$. Then $I^{\alpha}(\eta, D \eta)=-2\left(\eta, D^{\perp} \zeta^{\alpha}\right)$, so that

$$
\int_{0}^{\tau} k_{\alpha \beta} I^{\alpha}(\eta, D \eta) I^{\beta}(\eta, D \eta) d t=4 \int_{0}^{\tau} k_{\alpha \beta}\left(\eta, D^{\perp} \zeta^{\alpha}\right)\left(\eta, D^{\perp} \zeta^{\beta}\right) d t .
$$


Finally,

$$
\begin{aligned}
h(\eta, \eta)=\int_{0}^{\tau}\left(\left(D^{\perp} \eta, D^{\perp} \bar{\eta}\right)+k_{\alpha \beta}\left(\eta, D^{\perp} \zeta^{\alpha}\right)\left(\eta, D^{\perp} \zeta^{\beta}\right)+(U \eta, \bar{\eta})\right) d t & \\
& =h^{\perp}(\eta, \eta)+4 \int_{0}^{\tau} k_{\alpha \beta}\left(\eta, D^{\perp} \zeta^{\alpha}\right)\left(\eta, D^{\perp} \zeta^{\beta}\right) d t .
\end{aligned}
$$

It remains to substitute this into (5.17).

We consider the space

$$
Y^{0}=\{\xi \in X: I(\xi, D \xi) \equiv 0\} \subset Y .
$$

By (5.16) we have $\left.h\right|_{Y^{0}}=\left.h^{\perp} \circ \Pi\right|_{Y^{0}}$.

Assertion 5.16. We have

$$
Y^{0}+Z=\left\{\eta \in X: \int_{0}^{\tau} k_{\alpha \beta}\left(D^{\perp} \zeta^{\alpha}, \eta^{\beta}\right) d t=0\right\} .
$$

Thus, $Y^{0}+Z=X$ if and only if $D^{\perp} \zeta^{\alpha}=0$. Equivalently, $D Z \subset Z$. We set

$$
X_{0}^{\perp}=\Pi Y^{0}=\left\{\eta \in X^{\perp}: \int_{0}^{\tau} k_{\alpha \beta}\left(D^{\perp} \zeta^{\beta}, \eta\right) d t=0\right\} .
$$

Then $X_{0}^{\perp}$ has codimension $\leq k$ in $X^{\perp}$. We have $h^{\top} \geq h^{\perp}$ and $h^{\top}=h^{\perp}$ on $X_{0}^{\perp}$. Since ind $h^{\top}=$ ind $h$, it follows that

$$
\text { ind } h^{\perp} \leq \text { ind }\left.h\right|_{X} \leq \text { ind } h^{\perp}+k \text {. }
$$

5.5. The indices of $h$ and $h^{\perp}$. In this subsection we discuss the connection between the indices ind $h=$ ind $\left.h\right|_{Y}$ and ind $h^{\perp}$. Let $\widehat{G}^{-1} P: W \rightarrow W$ be the Poincaré map. As in Subsection 3.4 we assume that

$$
N=\operatorname{Ker}\left(\widehat{G}^{-1} P-I\right)^{2}=\left\{v \in W: \widehat{G}^{-1} P v-v \in V\right\}
$$

is a symplectic space and $V=\operatorname{Ker}\left(\widehat{G}^{-1} P-I\right)$ is a Lagrangian subspace of $N$. Let $w^{1}, \ldots, w^{k}$ be a basis in the space $V$ and let $q_{1}, \ldots, q_{k}$ be a basis in the Lagrangian complement of $V$ in $N$. We define matrices $A=\left(a_{\alpha \beta}\right)$ and $A^{\perp}=\left(a_{\alpha \beta}^{\perp}\right)$ by the formulae

$$
a_{\alpha \beta}=s_{\alpha \delta} \kappa^{\delta \varepsilon} s_{\varepsilon \beta}-s_{\alpha \delta}, \quad a_{\alpha \beta}^{\perp}=s_{\alpha \beta}-\bar{k}_{\alpha \beta} .
$$

Recall the nondegeneracy conditions of Subsection 3.4

Condition A. The symmetric matrix $K_{i}=\left(k_{i}^{\alpha \beta}\right), k_{i}^{\alpha \beta}=\left\langle B_{i} w_{i}^{\alpha}, w_{i+1}^{\beta}\right\rangle$, is nondegenerate for all $i$.

Condition B. The matrix

$$
\bar{K}=\sum_{i=1}^{n} K_{i}^{-1}=\left(\bar{k}_{\alpha \beta}\right), \quad \bar{k}_{\alpha \beta}=\sum_{i=1}^{n} k_{\alpha \beta i}
$$

is nondegenerate.

Condition C. The matrix $A^{\perp}=\left(a_{\alpha \beta}^{\perp}\right)$ is nondegenerate.

Definition 5.17. We say that $\gamma$ is nondegenerate $\bmod V$ if the nondegeneracy Conditions $\mathrm{A}, \mathrm{B}$, and $\mathrm{C}$ hold.

Theorem 5.18. Suppose that $\gamma$ is nondegenerate $\bmod V$. Then

$$
\text { ind } h-\text { ind } h^{\perp}=\text { ind } A-\text { ind } A^{\perp} \text {. }
$$


See the proof in [1].

Since the form $\left.h\right|_{\widehat{Z}}$ is positive definite, as in the proof of Corollary 3.17 we obtain the following.

Corollary 5.19. Suppose that $\operatorname{det} A \neq 0$. Then $(-1)^{\operatorname{ind} h}=(-1)^{\operatorname{ind} h^{\perp}+\operatorname{ind} b}$.

5.6. Example: Autonomous systems. Consider the special case of autonomous Lagrangian systems. The system of variational equations of a $g$-periodic trajectory $\gamma$ has a $G$-periodic solution $\zeta(t)=\dot{\gamma}(t)$. We assume that $\gamma$ is nondegenerate in the autonomous sense (only two multipliers equal to 1 ) and there exists a family $\gamma_{\alpha}$ of $g$-periodic trajectories of period $\tau(\alpha)$ such that $\gamma_{0}=\gamma$ and $\tau(0)=\tau$. Let $E(\alpha)=\left.\mathscr{H}\right|_{\gamma_{\alpha}}$ and $A(\alpha)=\int_{\gamma_{\alpha}}\langle p, d x\rangle$ be the energy and the Maupertuis action of the trajectory $\gamma_{\alpha}$.

Lemma 5.20. Suppose that $d E / d \tau \neq 0$. Then

$$
(-1)^{\operatorname{ind} b}=-\operatorname{sign}\left(\tau^{\prime}(\alpha) E^{\prime}(\alpha)\right)=-\operatorname{sign}\left(\frac{d E}{d \tau}\right) .
$$

Proof. The union of the trajectories $\gamma_{\alpha}$ in the phase space $T M \cong T^{*} M$ is a symplectic cylinder $\Sigma$. Restricting the Hamiltonian system to $\Sigma$ we obtain an integrable Hamiltonian system with one degree of freedom and with Hamiltonian $H(\vartheta, I)=E(I)$, where $\vartheta \in$ $\mathbb{R} / \mathbb{Z}, I \in \mathbb{R}$. We can assume that $\alpha=I$ and $\gamma_{\alpha}(t)=\left(E^{\prime}(\alpha) t, \alpha\right)$. Then $\tau(\alpha)=1 / \nu(\alpha)$, where the frequency $\nu(\alpha)$ is equal to $E^{\prime}(\alpha)$. We have $w=\left(\begin{array}{l}1 \\ 0\end{array}\right)$ and $v=\left(\begin{array}{l}0 \\ 1\end{array}\right)$. The monodromy matrix of the trajectory $\gamma_{\alpha}$ is

$$
\widehat{G}^{-1} P=\widehat{G}^{-1} P_{\alpha}=\left(\begin{array}{cc}
1 & \nu^{\prime} / \nu \\
0 & 1
\end{array}\right)
$$

Hence,

$$
\widehat{G}^{-1} P v=\left(\begin{array}{c}
\nu^{\prime} / \nu \\
1
\end{array}\right)
$$

so that $s=\omega\left(\widehat{G}^{-1} P v, v\right)=\nu^{\prime} / \nu=-\tau^{-2} d \tau / d E$.

Notation. Set ind $\gamma=$ ind $h(\gamma)$.

Assertion 5.21. Suppose that a g-periodic trajectory $\gamma$ has exactly 2 multipliers equal to 1. Suppose that $\sigma(-1)^{m+i n d} \gamma d E / d \tau<0$. Then $\gamma$ has a real multiplier $\rho>1$.

Proof. Since $\sigma=\sigma^{\perp}$, by Corollary 5.19 and Lemma 5.20 we have

$$
(-1)^{\text {ind } h^{\perp}}=-\operatorname{sign}\left(\frac{d E}{d \tau}\right)(-1)^{\text {ind } \gamma}
$$

The dimension of the reduced system is equal to $m^{\perp}=m-1$. Therefore,

$$
\sigma(-1)^{m^{\perp}+\text { ind } h^{\perp}}=\sigma(-1)^{m-1+\text { ind } \gamma}\left(-\operatorname{sign}\left(\frac{d E}{d \tau}\right)\right)=-1 .
$$

If we apply Corollary 4.35 to the reduced Hill formula (4.26), we find that there exists a multiplier $\rho>1$.

Example 5.22. Suppose that a material point in $\mathbb{R}^{m}$ is moving in a potential field with a homogeneous potential

$$
V(\lambda x)=\lambda^{k} V(x), \quad \lambda>0, k(k-2) \neq 0 .
$$

Suppose that $g: \mathbb{R}^{m} \rightarrow \mathbb{R}^{m}$ is a diffeomorphism preserving the Lagrangian. We introduce generalized spherical coordinates $\left(r, \phi_{1}, \ldots, \phi_{m-1}\right), g(x)=\left(g_{r}(x), g_{\phi_{1}}(x), \ldots, g_{\phi_{m-1}}(x)\right)$ 
in $\mathbb{R}^{m}$. Then $V(x)$ can be represented in the form

$$
\begin{gathered}
V(x)=r^{k} f\left(\phi_{1}, \ldots, \phi_{m-1}\right), \quad g_{r}(x)=r, \\
f\left(g_{\phi_{1}}(x), \ldots, g_{\phi_{m-1}}(x)\right)=f\left(\phi_{1}, \ldots, \phi_{m-1}\right) .
\end{gathered}
$$

Let $\gamma$ be a $g$-periodic solution with energy $E$ such that $\gamma(t+\tau)=g \gamma(t)$ for all $t \in \mathbb{R}$. Then $\gamma_{\lambda}(t)=\gamma\left(\lambda^{k / 2-1} t\right)$ is a $g$-periodic solution with 'period' $\tau(\lambda)=\lambda^{1-k / 2} \tau$ and energy $E(\lambda)=\lambda^{k} E$. Therefore,

$$
\frac{d E(\lambda)}{d \tau(\lambda)}=\frac{2 k}{k-2}\left(\frac{\tau(\lambda)}{\tau}\right)^{(k+2) /(k-2)} E .
$$

Hence, by Lemma 5.20 ,

$$
(-1)^{\text {ind } b}=\operatorname{sign}\left(\frac{2-k}{k}\right) .
$$

Consider the problem of the motion of a material point in $\mathbb{R}^{m}$ in a force field with a homogeneous potential of degree $k$, where $k(k-2) \neq 0$. The equations $\sigma=1$, (5.24) and Assertion 5.21 yield the following.

Proposition 5.23. Suppose that a g-periodic trajectory $\gamma$ has exactly 2 multipliers equal to 1 . Suppose that $(-1)^{m+\operatorname{ind} \gamma}(k-2) / k<0$. Then $\gamma$ has a real multiplier $\rho>1$.

\section{REFERENCES}

[1] S. V. Bolotin and D. V. Treshchëv, Hill's formula, Uspekhi Mat. Nauk 65 (2010), no. 2, 3-70; English transl., Russian Math. Surveys 65 (2010), no. 2, 191-257. MR2668800 (2012g:37158)

[2] G. W. Hill, On the part of the motion of the lunar perigee which is a function of the mean motions of the sun and moon, Acta Math. 8 (1886), no. 1, 1-36. MR.1554690

[3] A. Poincaré, Les méthodes nouvelles de la mécanique céleste. Vol. 1-3, Gauthier-Villars, Paris, 1892, 1893, 1899.

[4] R. S. MacKay and J. D. Meiss, Linear stability of periodic orbits in Lagrangian systems, Phys. Lett. A 98 (1983), no. 3, 92-94. MR721605 (85a:58031)

[5] D. V. Treshchëv, On the problem of the stability of the periodic trajectories of a Birkhoff billiard, Vestnik Moskov. Univ. Ser. I Mat. Mekh. 1988 (1988), no. 2, 44-50; English transl., Mosc. Univ. Mech. Bull. 43 (1988), no. 2, 28-36. MR938065 (89k:58246)

[6] S. V. Bolotin, The Hill determinant of a periodic orbit, Vestn. Mosk. Univ., Ser. I Mat. Mekh. 1988 (1988), no. 3, 30-34; English transl., Mosc. Univ. Mech. Bull. 43 (1988), no. 3, 7-11. MR966862 (89i:58030)

[7] V. V. Kozlov and D. V. Treshchëv, Billiards. A genetic introduction to the dynamics of systems with impacts, Moscow Univ., Moscow, 1991; English transl., Transl. Math. Monographs, vol. 89, Amer. Math. Soc., Providence, RI, 1991. MR.1118378 (93k:58094a)

[8] V. V. Kozlov, On the mechanism of stability loss, Differentsial'nye Uravneniya 45 (2009), no. 4, 496505; English transl., Differential Equations 45 (2009), no. 4, 510-519, MR2596746 (2011c:34128)

[9] X. Hu and P. Wang, Conditional Fredholm determinant for the S-periodic orbits in Hamiltonian systems, J. Funct. Anal. 261 (2011), no. 11, 3247-3278. MR2835998

[10] V. V. Kozlov, Spectral properties of operators with polynomial invariants in real finite-dimensional spaces, Trudy Mat. Inst. Steklova 268 (2010), 155-167; English transl., Proc. Steklov Inst. Math. 268 (2010), no. 1, 148-160. MR2724341(2012b:47007)

[11] C. Liu and Y. Long, Iterated index formulae for closed geodesics with applications, Sci. China Ser. A 45 (2002), no. 1, 9-28. MR.1894955 (2003c:53051)

[12] Y. Long, Index theory for symplectic paths with applications, Progress in Math., vol. 207, Birkhäuser, Basel, 2002. MR1898560 (2003d:37091)

[13] A. P. Veselov, Integrable mappings, Uspekhi Mat. Nauk, 46 (1991), no. 5, 3-45; English transl., Russian Math. Surveys, 46 (1991), no. 5, 1-51. MR1160332(93e:58096)

[14] D. McDuff and D. Salamon, Introduction to symplectic topology, Clarendon Press, Oxford Univ. Press, New York, 1998. MR.1702941 (2000e:53099)

[15] D. V. Treshchëv, The connection between the Morse index of a closed geodesic and its stability, Trudy Sem. Vektor. Tenzor. Anal. 23 (1988), 175-189. (Russian) MR1041280 (91e:58044) 
[16] V. I. Arnold, V. V. Kozlov, and A. I. Neřshtadt, Mathematical aspects of classical and celestial mechanics, URSS, Moscow, 2011; English transl., Encyclopaedia of Mathematical Sciences, vol. 3. Springer-Verlag, Berlin, 2006. MR2269239 (2008a:70001)

[17] V. I. Arnol'd, Mathematical methods of classical mechanics, URSS, Moscow, 2003; English transl. of 2nd ed., Graduate Texts in Mathematics, vol. 60, Springer-Verlag, New York, 1989. MR.997295 (90c:58046)

[18] J. Milnor, Morse theory, Annals of Math. Studies, vol. 51, Princeton Univ. Press, Princeton, N.J, 1963. MR0163331 (29:634)

[19] X. Hu and S. Sun, Index and stability of symmetric periodic orbits in Hamiltonian systems with application to figure-eight orbit, Comm. Math. Phys. 290 (2009), no. 2, 737-777. MR2525637 (2010h:37140)

[20] X. Hu and S. Sun, Morse index and stability of elliptic Lagrangian solutions in the planar three-body problem, Adv. Math. 223 (2010), no. 1, 98-119. MR.2563212 (2011e:37118)

[21] A. Chenciner and R. Montgomery, A remarkable periodic solution of the three-body problem in the case of equal masses, Ann. Math. (2) 152 (2000), no. 3, 881-901. MR1815704 (2001k:70010)

[22] D. Ferrario and S. Terracini, On the existence of collisionless equivariant minimizers for the classical n-body problem, Invent. Math. 155 (2004), no. 2, 305-362. MR2031430 (2005b:70010)

[23] S. Terracini and A. Venturelli, Symmetric trajectories for the $2 N$-body problem with equal masses, Arch. Ration. Mech. Anal. 184 (2007), no. 3, 465-493. MR2299759(2008f:70033)

[24] H. R. Dullin and J. D. Meiss, Stability of minimal periodic orbits, Phys. Lett. A 247 (1998), no. 3, 227-234. MR 1652774 (99f:58168)

[25] V. V. Kozlov, The problem of stability of two-link trajectories of a multidimensional Birkhoff billiard, Trudy Mat. Inst. Steklova 273 (2011), 212-230; English transl., Proc. Steklov Inst. Math. 273 (2011), no. 1, 196-213. MR2893547

[26] M. Bialy, Maximizing orbits for higher-dimensional convex billiards, J. Modern Dynam. 3 (2009), no. 1, 51-59. MR2481332(2009m:37180)

[27] G. D. Birkhoff, Dynamical systems. With an addendum by Jurgen Moser, Amer. Math. Soc. Colloq. Publ., vol. IX, Amer. Math. Soc., Providence, R.I. 1966. MR0209095 (35:1)

[28] Ya. G. Sinaŭ, Dynamical systems with elastic reflections. Ergodic properties of dispersing billiards, Uspekhi Mat. Nauk 25 (1970) no. 2, 141-192. (Russian) MR0274721 (43:481)

[29] S. Aubry and G. Abramovici, Chaotic trajectories in the standard map. The concept of antiintegrability, Phys. D 43 (1990), no. 2-3, 199-219. MR1067910 (91j:58100)

[30] R. S. MacKay and J. D. Meiss, Cantori for symplectic maps near the anti-integrable limit, Nonlinearity 5 (1992), no. 1, 149-160. MR1148793 (93b:58123)

[31] D. Treschev and O. Zubelevich, Introduction to the perturbation theory of Hamiltonian systems, Springer-Verlag, Berlin, 2010. MR2554208 (2011b:37116)

[32] S. Bolotin and R. MacKay, Multibump orbits near the anti-integrable limit for Lagrangian systems, Nonlinearity 10 (1997), no. 5, 1015-1029. MR.1473371 (98h:34090)

[33] W. Klingenberg, Lectures on closed geodesics, Grundl. Math. Wiss., vol. 230, Springer-Verlag, Berlin-New York, 1978. MR0478069 (57:17563)

[34] R. Bott, On the iteration of closed geodesics and Sturm intersection theory, Commun. Pure Appl. Math. 9 (1956), 171-206. MR0090730(19:859f)

Moscow State University

E-mail address: marsdavletshin@mail.ru

Translated by E. KHUKHRO

Originally published in Russian 